\title{
THE JACOBIAN CONJECTURE: REDUCTION OF DEGREE AND FORMAL EXPANSION OF THE INVERSE
}

BY HYMAN BASS, ${ }^{1}$ EDWIN H. CONNELL ${ }^{2}$ AND DAVID WRIGHT ${ }^{3}$

\section{CONTENTS}

\section{Introduction}

I. The Jacobian Conjecture

1. Statement of the Jacobian Problem; first observations

2. Some history of the Jacobian Conjecture

3. Faulty proofs

4. The use of stabilization and of formal methods

II. The Reduction Theorem

1. Notation

2. Statement of the Reduction Theorem

3. Reduction to degree 3

4. Proof of the Reduction Theorem

5. $T$-linearization and unipotent reduction

6. Nilpotent rank 1 Jacobians

III. The Formal Inverse

1. Notation

2. Abhyankar's Inversion Formula

3. The terms $G_{i}^{(d)}$

4. The tree expansion $G_{i}^{(d)}=\Sigma_{T}(1 / \alpha(T)) \Sigma_{f} P_{T, f}$

5. Calculations

References

\section{INTRODUCTION}

A mapping $F: \mathbf{C}^{n} \rightarrow \mathbf{C}^{n}, F(X)=\left(F_{1}(X), \ldots, F_{n}(X)\right)$, is a polynomial mapping if each $F_{i}$ is a polynomial. How do we recognize when such an $F$ is invertible? The question is unambiguous since, once $F$ is bijective, its set theoretic inverse is automatically polynomial (see Theorem 2.1). When $F$ is

Received by the editors January 9, 1982.

1980 Mathematics Subject Classification. Primary 13B25, 13B10, 13B15, 14E05, 14E07; Secondary $05 \mathrm{C} 05$.

'Partially supported by NSF Grant MCS 80-05802.

${ }^{2}$ Partially supported by NSF Grant MCS 77-28188.

${ }^{3}$ Partially supported by NSF Grant MCS $80-02844$. 
linear, nonvanishing of $\operatorname{det}(F)$ detects invertibility. Linearizing the general case leads us to consider the Jacobian $J(F)=\left(\partial F_{i} / \partial X_{j}\right)$. Invertibility of $F$ implies that of $J(F)$, i.e. that det $J(F) \in \mathbf{C}^{x}$. Conversely we have the Jacobian Conjecture:

$$
\operatorname{det} J(F) \in \mathbf{C}^{x} \Rightarrow F \text { is invertible. }
$$

The analogue in characteristic $p>0$ is false, already for $n=1, F(X)=X+$ $X^{p}$. The analytic analogue is likewise false, for example with $n=2, F_{1}=e^{X}$, $F_{2}=e^{-X} Y$. There is even an entire $F: \mathbf{C}^{2} \rightarrow \mathbf{C}^{2}$ with det $J(F)=1$ such that $F$ is injective, yet $F\left(C^{2}\right)$ misses a nonempty open set (see (1.1), Remark 10). In contrast, if $F: \mathbf{C}^{n} \rightarrow \mathbf{C}^{n}$ is polynomial, det $J(F)=1$, and $F$ is injective, then $F$ is invertible (see Theorem 2.1). Thus the Jacobian Conjecture must depend on properties specific to polynomials in characteristic zero.

The Jacobian Conjecture seems first to have been formulated by $\mathrm{O}$. $\mathrm{H}$. Keller in 1939. Aside from the trivial case $n=1$ it remains an open problem for all $n \geqslant 2$. In the late fifties and early sixties several faulty proofs appeared in print. A discussion of these is presented in $\S 3$ of Chapter I.

Chapter I aims to give a fairly complete representation of the state of the art on the Jacobian Conjecture, to serve as a historical account and as a reference for future research on the problem. $\$ 2$ surveys a number of the partial results. Notable among these is Moh's proof of the Jacobian Conjecture for $n=2$ when $\operatorname{deg}(F) \leqslant 100$ [Mo], and S. Wang's proof of it for all $n$ when $\operatorname{deg}(F) \leqslant 2$ [Wa]. Here $\operatorname{deg}(F)$ denotes the maximum degree of the polynomials $F_{1}, \ldots, F_{n}$.

Chapters II and III present a new approach to the Jacobian Conjecture, whose effect is to reduce it to a sort of combinatorial problem, hopefully susceptible to testing by computer.

In Chapter II it is shown that the Jacobian Conjecture will follow once it is shown for all $F=\left(F_{1}, \ldots, F_{n}\right)$ of the form $F_{i}=X_{i}-H_{i}$ where each $H_{i}$ is a cubic homogeneous polynomial, and the matrix $J(H)=\left(\partial H_{i} / \partial X_{j}\right)$ is nilpotent. (This contrasts temptingly with Wang's proof of the conjecture for quadratic $F$.)

An $F=X-H$ as above has an analytic inverse $G=\left(G_{1}, \ldots, G_{n}\right)$ near the origin: For each $i, G_{i}(X)$ is a power series such that $G_{i}(F)=X_{i}$. The Jacobian Conjecture asserts that these power series $G_{i}$ are polynomials. We present in Chapter III a formula of Abhyankar and Joni for the $G_{i}$. From this we derive a combinatorial expansion (indexed by rooted trees with $d$ vertices) for the homogeneous components $G_{i}^{(d)}$ of $G_{i}$. The hope is to show that $G_{i}^{(d)}=0$ for sufficiently large $d$. We initiate a (still inconclusive) method for showing this by induction on $e$, where $J(H)^{e}=0$.

The three chapters may be read essentially independently of one another.

This paper is a substantial revision of an earlier draft circulated in August, 1980. It owes much to numerous colleagues, who are acknowledged at the end of Chapter I.

\section{The Jacobian Conjecture}

1. Statement of the Jacobian Problem; first observations. Let $k$ be a commutative ring and $n \geqslant 0$ an integer. Write $k^{[n]}$ for the polynomial algebra $k\left[X_{1}, \ldots, X_{n}\right]$ in $n$ variables and $\mathbf{A}_{k}^{n}=\operatorname{Spec}\left(k^{[n]}\right)$, affine $n$-space over $k$. A 
morphism, or polynomial map, $F: \mathbf{A}_{k}^{n} \rightarrow \mathbf{A}_{k}^{n}$ can be identified with its sequence $F=\left(F_{1}, \ldots, F_{n}\right)$ of coordinate functions $F_{i} \in k^{[n]}$. The induced $k$-algebra endomorphism of $k^{[n]}$ is $\varphi_{F}: f \mapsto f(F)$, sending $X_{i}$ to $F_{i}$. The map $F \mapsto \varphi_{F}$ is an anti-isomorphism of monoids $\left(\varphi_{F} \circ \varphi_{G}=\varphi_{G} \circ F\right)$,

$$
\operatorname{End}\left(\mathbf{A}_{k}^{n}\right) \rightarrow \operatorname{End}\left(k^{[n]}\right)
$$

from $k$-scheme endomorphisms to $k$-algebra endomorphisms. Each $F$ as above defines a polynomial map $F: k^{n} \rightarrow k^{n}$ which determines $F$ whenever $k$ is, say, an infinite integral domain.

The automorphism group

$$
G A_{n}(k)=\operatorname{Aut}\left(\mathbf{A}_{k}^{n}\right)
$$

is here called the affine Cremona group; it is anti-isomorphic to $\mathrm{Aut}_{k \text {-alg }}\left(k^{[n]}\right)$. The group $G A_{n}$ is the nonlinear analogue of $G L_{n}$.

Given $F \in \operatorname{End}\left(\mathbf{A}_{k}^{n}\right)$ how can we recognize whether or not $F \in G A_{n}(k)$ ? A necessary condition is furnished by the Jacobian matrix

$$
J(F)=\left(\begin{array}{ccc}
D_{1} F_{1} & \cdots & D_{n} F_{1} \\
\vdots & & \vdots \\
D_{1} F_{n} & \cdots & D_{n} F_{n}
\end{array}\right) \quad\left(J(F)_{i, j}=D_{j} F_{i}\right),
$$

where $D_{j}=\partial / \partial X_{j}$. The Chain Rule gives

$$
J(G(F))=J(G)(F) \cdot J(F) .
$$

When $F$ is invertible, with inverse $G$, we have $G(F)=X=\left(X_{1}, \ldots, X_{n}\right)$ so $I=J(X)=J(G)(F) \cdot J(F)$, and we see that $J(F)$ is invertible. Thus

$$
F \in G A_{n}(k) \Rightarrow J(F) \in G L_{n}\left(k^{[n]}\right) .
$$

In this case $\operatorname{det} J(F)$ is a unit of $k^{[n]}$. When $k$ is reduced (without nonzero nilpotent elements) the units of $k^{[n]}$ are just the (constant) units $k^{\times}$of $k$.

The Jacobian Problem asks about the converse of (2), i.e. about the validity of the following "Jacobian Property":

$$
J P_{n}(k): \text { if } F \in \operatorname{End}\left(\mathbf{A}_{k}^{n}\right) \text { and } J(F) \text { is invertible then } F \text { is invertible. }
$$

We begin with some elementary observations and then give a historical account of the problem.

(1.1) REMARKS. Let $F \in \operatorname{End}\left(\mathbf{A}_{k}^{n}\right)$.

1. To show that $F$ is invertible we are always at liberty to replace $F$ by $G \circ F \circ H$ where $G, H \in G A_{n}(k)$. In particular we can replace $F$ by $T \circ F$ where $T$ is the translation $T=X-F(0)$, and so arrange that $F(0)=0$. If the linear term $F_{(1)}$ of $F$ is an invertible linear map (it corresponds to the matrix $J(F)(0)$ ) we can further replace $F$ by $F_{(1)}^{-1} \circ F$ to arrange that $F_{(1)}=X$, so that $F_{i}=X_{i}+$ terms of degree $\geqslant 2(i=1, \ldots, n)$.

2. Suppose that $F(0)=0$ and that $J(F)(0)$ is invertible. Then (Implicit Function Theorem) $F$ is formally invertible at the origin. In other words there 
is a formal inverse $G=\left(G_{1}, \ldots, G_{n}\right)$ defined by the conditions,

$$
\begin{aligned}
& G_{i} \in k^{[[n]]}=k\left[\left[X_{1}, \ldots, X_{n}\right]\right], \\
& G_{i}(F)=X_{i} \quad(i=1, \ldots, n) .
\end{aligned}
$$

(We give a formula for $G_{i}$ in Chapter III, Corollary (2.2).) In order that $F \in G A_{n}(k)$ it is necessary and sufficient that the power series $G_{i}$ be polynomials.

In any case the map $\varphi_{F}: f \mapsto f(F)$ defines an automorphism of $k^{[[n]]}$, and hence an injective endomorphism of $k^{[n]}$, i.e. $F_{1}, \ldots, F_{n}$ are "algebraically independent" over $k$. Invertibility of $F$ is equivalent to the condition $k\left[F_{1}, \ldots, F_{n}\right]=k\left[X_{1}, \ldots, X_{n}\right]$.

3. Suppose that $J(F)(0)$ is invertible. Let $k \subset K$ be an extension of scalars. Then

$$
F \in G A_{n}(k) \Leftrightarrow F \in G A_{n}(K) .
$$

In fact we may assume that $F(0)=0$ so that $F$ has a formal inverse $G=$ $\left(G_{1}, \ldots, G_{n}\right)$. Then $G_{i} \in k^{[[n]]}$ is a polynomial over $k$ iff it is one over $K$.

4. ("Lefschetz Principle"). $J P_{n}(\mathbf{C}) \Rightarrow J P_{n}(k)$ for every integral domain $k$ of characteristic 0 . In fact let $F \in \operatorname{End}\left(\mathbf{A}_{k}^{n}\right)$ with $J(F)$ invertible. Using Remark 1 we may assume that $F(0)=0$ and $J(F)(0)=I$. In this case det $J(F)=1$. Let $k_{0}$ be the subring of $k$ generated by all coefficients of $F_{1}, \ldots, F_{n}$. Then $k_{0}$ can be embedded in $\mathrm{C}$. By assumption $J P_{n}(\mathrm{C})$ we have $F \in G A_{n}(\mathrm{C})$, so by Remark 3 we have $F \in G A_{n}\left(k_{0}\right) \subset G A_{n}(k)$.

5. If $k$ is a field of characteristic $p>0$ then $J P_{n}(k)$ fails for all $n \geqslant 1$. Just take $F_{1}=X_{1}+X_{1}^{p}$ and $F_{i}=X_{i}$ for $i \geqslant 2$. Then $J(F)=I$ whereas $F$ can't be invertible since $F_{1}$ is not even an irreducible polynomial.

6. Suppose that $N$ is a nilpotent ideal of $k$, and put $\bar{k}=k / N$. Let $\bar{F}=\bar{k} \otimes_{k} F$ be the corresponding endomorphism of $\mathbf{A}_{\bar{k}}^{n}$. Then

$$
F \in G A_{n}(k) \Leftrightarrow \bar{F} \in G A_{n}(\bar{k}) .
$$

For the nonobvious implication, suppose that $\bar{F} \in G A_{n}(\bar{k})$. Let $C$ denote the $k$-module $k^{[n]} / \varphi_{F}\left(k^{[n]}\right)$. Since $\varphi_{F}=\bar{k} \otimes_{k} \varphi_{F}$ is an isomorphism we have $C / N C=0$, so $C=N C=N^{2} C=\cdots$. Since $N$ is nilpotent, $C=0$. Since $k^{[n]}$ is $k$-free, $\operatorname{Ker}\left(\varphi_{F}\right)$ is a $k$-linear direct summand of $k^{[n]}$. Therefore, again since $\varphi_{F}$ is an isomorphism, ${ }_{\bar{k}} \otimes_{k} \operatorname{Ker}\left(\varphi_{F}\right)=0$, and again as above, $\operatorname{Ker}\left(\varphi_{F}\right)=0$.

7. Suppose that $k$ is noetherian. Assume that $J(F)(0)$ is invertible. If for every homomorphism $k \rightarrow K$, where $K$ is a field, $K \otimes_{k} F \in G A_{n}(K)$, then $F \in G A_{n}(k)$.

We may assume that $F(0)=0$. Let $G$ be the formal inverse of $F($ Remark 2). Let $P$ be a prime ideal of $k$, and $K$ the field of fractions of $k / P$. By hypothesis, $K \otimes_{k} F$ is invertible, so that $G$ is a polynomial map $\bmod P$. Therefore $G$ is a polynomial map modulo the intersection $N$ of the (finitely many) minimal primes of $k$. Since $N$ is nilpotent the invertibility of $F$ follows from Remark 6.

8. $J P_{n}(\mathrm{C}) \Rightarrow J P_{n}(k)$ for every commutative ring $k$ which is $\mathbf{Z}$-torsion free, or, more generally, in which the $\mathbf{Z}$-torsion elements are nilpotent.

Indeed, let $F \in \operatorname{End}\left(\mathbf{A}_{k}^{n}\right)$ with $J(F)$ invertible. By Remark 1 we may assume that $F(0)=0$ and $J(F)(0)=I$. Thanks to Remark 3 we may replace $k$ by the subring generated by all coefficients of $F$ and of det $J(F)^{-1}$ and so assume 
that $k$ is noetherian. Then its nilradical is nilpotent, so, by Remark 6, we may replace $k$ by $k / N$, where $N$ is the (nilpotent) ideal of Z-torsion elements, and so assume that $k$ embeds in $K=\mathbf{Q} \otimes_{\mathrm{z}} k$. By Remark 3 again, we may replace $k$ by $K$. Then the assertion follows from Remarks 4 and 7 .

9. One can generalize the Jacobian Problem to morphisms $F=\left(F_{1}, \ldots, F_{m}\right)$ from $\mathbf{A}_{k}^{n}$ to $\mathbf{A}_{k}^{m}$ (each $F_{i}$ being in $k^{[n]}$ ), by asking when $F$ admits a left inverse $G$. From the formula $I_{n}=J(G(F))=J(G)(F) \cdot J(F)$ we see that a necessary condition is that $J(F)$ be left invertible. That this condition is not sufficient is shown by the following example, over any $k$. Take $F: \mathbf{A}_{k}^{1} \rightarrow \mathbf{A}_{k}^{2}$ defined by $F_{1}(T)=T^{2}-T$ and $F_{2}(T)=T^{3}-T^{2}$. Then $F(0)=F(1)=(0,0)$, so $F$ is not an embedding, whereas

$$
J(F)=\left(\begin{array}{c}
2 T-1 \\
3 T^{2}-2 T
\end{array}\right)
$$

has the left inverse $((6 T-1),-4)$, so that $F$ is an immersion.

10. The analytic analogue of the Jacobian Property fails completely, already for $n=2$. Taking $F_{1}=e^{X}$ and $F_{2}=Y e^{-X}$ we have det $J(F)=1$ everywhere. The fiber of $F$ through $(x, y)$ is $(x+\mathbf{Z} 2 \pi i, y)$, and the image of $F: \mathbf{C}^{2} \rightarrow \mathbf{C}^{2}$ excludes exactly the axis $X=0$. Even worse, there is an example $F=\left(F_{1}, F_{2}\right)$ : $\mathbf{C}^{2} \rightarrow \mathbf{C}^{2}$ of Fatou and Bieberbach (cf. [B $+\mathbf{M}$, Chapter III, §1]) such that $F_{1}$ and $F_{2}$ are entire, det $J(F)=1$ everywhere, $F$ is injective, and yet $\mathbf{C}^{2}-F\left(\mathbf{C}^{2}\right)$ contains a nonempty open set.

The degree of $F \in \operatorname{End}\left(\mathbf{A}_{k}^{n}\right)$ is defined to be

$$
\operatorname{deg}(F)=\max _{i} \operatorname{deg}\left(F_{i}\right)
$$

where the latter refers to degrees as polynomials in $X_{1}, \ldots, X_{n}$.

(1.2) Proposition. Let $k_{0}$ be a subring of $\mathbf{Q}$, and let $n, d$ be integers $\geqslant 1$. Let $C$ denote the class of all pairs $(k, F)$ where $k$ is a commutative $k_{0}$-algebra, $F \in \operatorname{End}\left(\mathbf{A}_{k}^{n}\right), \operatorname{deg}(F) \leqslant d$, and $\operatorname{det} J(F) \in k^{\times}$. Assume that whenever $(k, F)$ $\in C$ and $k$ is a field then $F$ is invertible. Then there is a constant $\delta=\delta\left(k_{0}, n, d\right)$ such that, for all $(k, F) \in C, F$ is invertible and the inverse of $F$ has degree $\leqslant \delta$.

Note that $k_{0}$ is obtained from $\mathbf{Z}$ by inverting some collection of primes.

Let $\mathfrak{M}$ denote the set of monomials of degree $\leqslant d$ in $X_{1}, \ldots, X_{n}$. Let $A$ denote the $k_{0}$-algebra of polynomials in indeterminates $c_{i, M}(i=1, \ldots, n$; $M \in \mathfrak{M})$. Put $\bar{F}=\left(\bar{F}_{1}, \ldots, \bar{F}_{n}\right)$ where $\bar{F}_{i}=\Sigma_{M \in \mathfrak{R}} c_{i, M} M \in A^{[n]}$. Let $D=$ $\operatorname{det} J(\bar{F}) \in A^{[n]}$, and let $J$ denote the ideal of $A$ generated by all coefficients of $D-1$ as a polynomial in $X_{1}, \ldots, X_{n}$. Put $B=A / J$ and $\tilde{F}=B \otimes_{A} \bar{F}$. Thus $(B, \tilde{F})$ is the generic element of $C$ subject to the condition det $J(\tilde{F})=1$. Since $B$ is noetherian it follows from Remark 7 above and our hypothesis that $\tilde{F}$ is invertible. Let $\tilde{G}$ denote its inverse, and put $\delta=\operatorname{deg}(\tilde{G})$. Let $(k, F) \in C$. If $u=\operatorname{det} J(F) \in k^{\times}$then we can replace $F_{1}$ by $u^{-1} F_{1}$ without changing the degree of $F$, or of its eventual inverse, and so arrange that det $J(F)=1$. Then the generic property of $(B, \tilde{F})$ implies that $F=k \otimes_{B} \tilde{F}$ for a unique homomorphism $B \rightarrow k$. It follows that $F$ is invertible with inverse $G=k \otimes_{B} \tilde{G}$ of degree $\leqslant \delta$. 
(1.3) Remarks. 1. Let $k$ be a field of characteristic $\neq 2$ and let $F \in \operatorname{End}\left(\mathbf{A}_{k}^{n}\right)$ be a quadratic map $(\operatorname{deg}(F) \leqslant 2)$ with $J(F)$ invertible. Then $S$. Wang [Wa] (see (2.4) below) has shown that $F$ is invertible, and he conjectured that its inverse $G$ has degree $\leqslant 2^{n-1}$. It follows from Wang's theorem that the hypothesis of Proposition (1.2) is satisfied for $k_{0}=\mathbf{Z}[1 / 2]$, all $n$, and $d=2$. Thus (1.2) gives a universal bound $\delta$ for $\operatorname{deg}(G)$. We shall see below (Corollary (1.4)) that in fact Wang's Conjecture is true.

2. When $k$ is not reduced the condition det $J(F) \in k^{\times}$in (1.2) is stronger than invertibility of $J(F)$, and the analogous proposition is false if we use the weaker condition. For example suppose $a \in k$ satisfies $a^{d} \neq 0, a^{d+1}=0$. Take $n=1$ and $F=X-a X^{2}$. Then $J(F)=1-2 a X \in k[X]^{\times}$. If $G(F)=X$ then $J(G)(F) \cdot J(F)=1$, so $J(G)\left(X-a X^{2}\right)=(1-2 a X)^{-1}=\sum_{i=0}^{d}(2 a X)^{i}$ has degree $\leqslant 2 \cdot \operatorname{deg} J(G)$. Thus $\operatorname{deg}(G) \geqslant(d / 2)+1$ whereas $\operatorname{deg}(F)=2$.

3. Let $k$ be a field and let $F \in \operatorname{End}\left(\mathbf{A}_{k}^{n}\right)$ be of degree $d \geqslant 1$. Write $F_{i}=\sum_{r=0}^{d} F_{i(r)}$, where each $F_{i(r)}$ is homogeneous of degree $r$, and put $f_{i}=$ $\sum_{r=0}^{d} X_{0}^{d-r} F_{i(r)}$. This is a homogeneous form of degree $d$ in $k\left[X_{0}, X_{1}, \ldots, X_{n}\right]$. Putting $f_{0}=X_{0}^{d}$, the sequence $f=\left(f_{0}, f_{1}, \ldots, f_{n}\right)$ defines a rational map

$$
f: \mathbf{P}_{k}^{n}-\rightarrow \mathbf{P}_{k}^{n}
$$

which extends $F$ on $\mathbf{A}_{k}^{n}$ (identified with the locus $X_{0} \neq 0$ ). If $\operatorname{deg}\left(F_{i}\right)=d$ then $X_{0}$ doesn't divide $f_{i}$, so g.c.d. $\left(f_{0}, f_{i}\right)=1$. If $F$ is invertible then $f$ is birational. It follows therefore from Theorem (1.5) below that if $F$ is invertible with inverse $G$ then $\operatorname{deg}(G) \leqslant \operatorname{deg}(F)^{n-1}$. We state this corollary now, before formulating the theorem.

(1.4) CoRollaRY. If $k$ is a field and $F \in G A_{n}(k)$ then $\operatorname{deg}\left(F^{-1}\right) \leqslant \operatorname{deg}(F)^{n-1}$.

The following result was communicated to us by Ofer Gabber, to whom we are grateful for permission to reproduce it here. He attributed it to an unrecalled colloquium lecturer at Harvard. ${ }^{4}$

Let $k$ be a field and let $f: \mathbf{P}^{n} \rightarrow \rightarrow \mathbf{P}^{n}$ be a rational map (where $\mathbf{P}^{n}=\mathbf{P}_{k}^{n}$ ); it is given by a sequence $\left(f_{0}, f_{1}, \ldots, f_{n}\right)$ of homogeneous forms of the same degree $d$, unique up to a factor from $k\left[X_{0}, \ldots, X_{n}\right]$. We can assume that g.c.d. $\left(f_{0}, \ldots, f_{n}\right)=1$, and then we define $\operatorname{deg}(f)=d=\operatorname{deg}\left(f_{i}\right)$ for all $i$. The domain of definition of $f$ is

$$
\operatorname{dom}(f)=\mathbf{P}^{n}-V\left(f_{0}, \ldots, f_{n}\right)
$$

(1.5) TheOREM. Let $f: \mathbf{P}^{n} \rightarrow \rightarrow \mathbf{P}^{n}$ be a birational map with inverse $f^{-1}$. Then

$$
\operatorname{deg}\left(f^{-1}\right) \leqslant \operatorname{deg}(f)^{n-1} .
$$

We sketch here the proof indicated by $O$. Gabber. Let $Z_{f}$ denote the union of the irreducible components of dimension $>0$ of all of the fibers of

\footnotetext{
${ }^{4}$ Since writing this paper John Tyrrell (Kings College, Univ. of London) has indicated that this result was "well known" to the classical geometers. Tyrrell also knew, but never published, the "reduction to degree 3" of Proposition (3.1) in Chapter II below.
} 
$f: \operatorname{dom}(f) \rightarrow \mathbf{P}^{n}$; this is closed in $\operatorname{dom}(f)$. Let $U_{f}=\operatorname{dom}(f)-Z_{f}$. Then $f$ induces an isomorphism

$$
\bar{f}: \stackrel{U_{f}}{\cong} U_{f^{-1}}
$$

with $\bar{f}^{-1}=\overline{f^{-1}}$. Choose a hyperplane

$$
H_{u}=V\left(\sum_{i} u_{i} X_{i}\right) \subset \mathbf{P}^{n}
$$

containing no irreducible component of $f\left(Z_{f}\right)$ and so that,

$$
\begin{aligned}
& D=\overline{f^{-1}\left(H_{u}\right)}=V\left(\Sigma_{i} u_{i} F_{i}\right) \text { contains no irreducible compo- } \\
& \text { nents of } Z_{f} \text {. }
\end{aligned}
$$

Then $H_{u}^{0}=H_{u} \cap U_{f^{-1}}$ is irreducible and we have an isomorphism $\bar{f}: D \cap U_{f} \rightarrow$ $H_{u}^{0}$. It follows that

$$
D=\overline{\bar{f}^{-1}\left(H_{u}^{0}\right)}
$$

and that $\Sigma_{i} u_{i} F_{i}$ is an irreducible polynomial.

Let $H_{2}, \ldots, H_{n}$ be a general system of hyperplanes such that $D \cap H_{2}$ $\cap \cdots \cap H_{n}$ is finite and contained in $U_{f}$; these exist because of $(*)$. Then

$$
\begin{aligned}
\operatorname{deg}(f) & =\operatorname{deg}(D)=\operatorname{deg}\left(D \cdot H_{2} \cdots H_{n}\right) \\
& =\operatorname{deg}\left(\bar{f}^{-1}\left(H_{u}^{0}\right) \cdot\left(\left.H_{2}\right|_{U_{f}}\right) \cdots\left(\left.H_{n}\right|_{U_{f}}\right)\right) \\
& =\operatorname{deg}\left(H_{u}^{0} \cdot \bar{f}\left(\left.H_{2}\right|_{U_{f}}\right) \cdots \bar{f}\left(\left.H_{n}\right|_{U_{f}}\right)\right) .
\end{aligned}
$$

If $H_{i}=V\left(\Sigma_{j} a_{i j} X_{j}\right)$ and $f^{-1}$ is given by $\left(g_{0}, \ldots, g_{n}\right)$ then $\bar{f}\left(H_{i} \cap U_{f}\right)=D_{i} \cap$ $U_{f^{\prime}}$ where $D_{i}=V\left(\Sigma_{j} a_{i j} g_{j}\right)$. The 0 -cycle $H_{u}^{0} \cdot \bar{f}\left(\left.H_{2}\right|_{U_{f}}\right) \cdots \bar{f}\left(\left.H_{n}\right|_{U_{f}}\right)$ can be written as $\left.Z\right|_{U_{f}}$ where $Z=\left(H_{u} \cdot D_{2} \cdots D_{n}\right)_{p}$, and the subscript $p$ designates "proper intersection," defined as follows.

If $A$ and $B$ are cycles of $\mathbf{P}^{n}$ of codimensions $a$ and $b$ respectively then $(A \cdot B)_{p}$ denotes the sum of the codimension $a+b$ components of $|A| \cap|B|$ with coefficients given by intersection theory; one disregards components of codimension $<a+b$. This product is associative on effective cycles. Let $A$ and $B$ be effective. If they intersect properly then $(A \cdot B)_{p}=(A \cdot B)$ and $\operatorname{deg}(A \cdot B)=\operatorname{deg}(A) \cdot \operatorname{deg}(B)$. If they intersect nonproperly then $\operatorname{deg}(A \cdot B)_{p}$ $<\operatorname{deg}(A) \cdot \operatorname{deg}(B)$.

Now returning to the argument above we have

$$
\operatorname{deg}(f)=\operatorname{deg}\left(\left.Z\right|_{U_{f}}\right) \leqslant \operatorname{deg}(Z) \leqslant \operatorname{deg}\left(H_{u}\right) \cdot \operatorname{deg}\left(D_{2}\right) \cdots \operatorname{deg}\left(D_{n}\right) .
$$

Since $\operatorname{deg}\left(H_{u}\right)=1$ and $\operatorname{deg}\left(D_{i}\right)=\operatorname{deg}\left(f^{-1}\right)(i=2, \ldots, n)$ we conclude that $\operatorname{deg}(f) \leqslant \operatorname{deg}\left(f^{-1}\right)^{n-1}$. Replacing $f$ by $f^{-1}$ we obtain the theorem.

Let $k$ be a field. Put $E=\operatorname{End}\left(\mathbf{A}_{k}^{n}\right), J=\{F \in E \mid \operatorname{det} J(F)=1\}$, and $G=J$ $\cap G A_{n}(k)$. The Jacobian Property is equivalent to the condition $J=G$. For 
each $d \geqslant 1$ let $E_{(d)}=\{F \in E \mid \operatorname{deg}(F) \leqslant d\}, J_{(d)}=J \cap E_{(d)}$, and $G_{(d)}=G \cap$ $E_{(d)}$. Then $E_{(d)} \stackrel{(d)}{=}\left(k_{(d)}^{[n]}\right)^{n}$, where the space $k_{(d)}^{[n]}$ of polynomials of degree $\leqslant d$ has dimension $\left(\begin{array}{c}n+d \\ n\end{array}\right)$. Clearly $J_{(d)}$ is a closed subvariety of $E_{(d)}$. What is less obvious is that:

(1.6) COROLlaRY. $G_{(d)}$ is a closed subvariety of $E_{(d)}$.

Each $F \in J_{(d)}$ can be uniquely factored as $T \circ F^{\prime}$ where $T$ is a translation and $F^{\prime}(0)=0$. Let $H$ be the formal inverse of $F^{\prime}$, and write $H=\Sigma_{r \geqslant 0} H_{(r)}$ where the components of $H_{(r)}$ are all homogeneous of degree $r$. According to Corollary (1.4) we have $F \in G_{(d)} \Leftrightarrow H_{(r)}=0$ for all $r \geqslant d^{n-1}$. Since the coefficients of each $H_{(r)}$ are Z-polynomial in the coefficients of $F$, the corollary is proved.

(1.7) Remark. Corollary (1.6) gives a possible approach to the Jacobian Conjecture. We have $G_{(d)} \subset J_{(d)} \subset E_{(d)}$. To show that $G_{(d)}=J_{(d)}$ it would suffice to show, if possible, that $\operatorname{dim} G_{(d)}=\operatorname{dim} J_{(d)}$ and that $J_{(d)}$ is irreducible.

2. Some history of the Jacobian Conjecture. Let $F: \mathbf{A}_{k}^{n} \rightarrow \mathbf{A}_{k}^{n}$ be a morphism with $J(F)$ invertible. The Jacobian Conjecture claims then that $F$ is invertible in the case $k=\mathbf{C}$, or, equivalently, for any field of characteristic zero (Remark (1.1)4). It was apparently first formulated by O. H. Keller [K] in 1939. Keller curiously considers $F$ with integer coefficients. He verifies the conjecture in the birational case, i.e. when $F$ has an inverse formed of rational functions, i.e. $k(F)=k(X)$ (cf. Theorem (2.1) below).

For $n=1$ the conjecture holds trivially. (Since $F^{\prime}(X)=a \neq 0, F(X)=a X$ $+b$, and $X=a^{-1}(F-b)$.) For $n \geqslant 2$ the conjecture remains open to the best of our knowledge.

The case $n=2$ is discussed in detail in Abhyankar's Tata Lecture Notes [Ab1], where he proves the galois case: $k(X)$ is galois over $k(F)$. He also shows that the conjecture is equivalent to the curves $F_{i}=0$ having only one point at infinity in $\mathbf{P}^{2}$. He shows that they have at most two points at infinity, a result also proved by Makar-Limanov [M-L]. Nakai and Baba $[\mathbf{N}+\mathbf{B}]$, generalizing a result of Magnus [Ma], prove the conjecture when one of $d_{i}=\operatorname{deg}\left(F_{i}\right)$ is prime, or 4 , or if $d_{1}=2 p \geqslant d_{2}$ with $p$ an odd prime. Moreover Moh [Mo], using characteristic pairs and a computer search, has proved the conjecture when $d_{1}, d_{2}$ are $\leqslant 100$. Wright [Wr1] has shown that $F$ is invertible iff $J(F)$ is a product of elementary and diagonal matrices in $G L_{2}\left(k^{[2]}\right)$. Other approaches to the case $n=2$ are discussed in Vitushkin [V], Fridman [F], and Razar [R]. An interesting discussion of the case $n=2$, with connections to differential equations, is given by Meisters [Me].

For general $n$, many of the partial results are summarized by the following theorem.

(2.1) THEOREM. Let $k$ be a field and let $F: \mathbf{A}_{k}^{n} \rightarrow \mathbf{A}_{k}^{n}$ be a morphism with $J(F)$ invertible. Consider the following conditions.

(a) $F$ is invertible, i.e. $k[F]=k[X]$.

(b) $F$ is birational, i.e. $k(F)=k(X)$.

(c) $F: k^{n} \rightarrow k^{n}$ is injective.

(d) The integral closure $\overline{k[F]}$ of $k[F]$ in $k[X]$ is unramified over $k[F]$. 
(e) $k[X]$ is a finitely generated $k[F]$-module.

(e') $F$ is proper.

(f) $k[X]$ is a projective $k[F]$-module.

(g) $k(X)$ is galois over $k(F)$.

If $\operatorname{char}(k)=0$ the conditions are all equivalent. In general we have
(g) $\Leftarrow$
(a) $\Leftrightarrow$
(b) $\Leftrightarrow$
(d) $\Leftarrow$
(e) $\Leftrightarrow\left(\mathrm{e}^{\prime}\right) \Leftrightarrow$

except that $k$ should be infinite for $(\mathrm{c}) \Rightarrow(\mathrm{b})$.

The implications (d) $\Rightarrow$ (a) and $(\mathrm{g}) \Rightarrow$ (a) invoke the simple connectivity of $\mathbf{C}^{n}$, whose analogue in characteristic $p>0$ is false (Artin-Schreier coverings). The situation in characteristic $p$ has recently been clarified by Nousiainen.

(2.2) THEOREM (NOUSIAINEN [N1]). Let $k$ be a field of characteristic $p>0$ and let $F: \mathbf{A}_{k}^{n} \rightarrow \mathbf{A}_{k}^{n}$ be a morphism. The following conditions are equivalent.

(1) $J(F)$ is invertible.

(2) $k[X]=k\left[X^{p}, F\right]\left(\right.$ i.e. $\left.k\left[X_{1}, \ldots, X_{n}\right]=k\left[X P, \ldots, X_{n}^{p}, F_{1}, \ldots, F_{n}\right]\right)$

(3) The monomials $F^{q}=F_{1}^{q_{1}} \cdots F_{n}^{q_{n}}\left(0 \leqslant q_{i}<p\right)$ form a free basis of the $k\left[X^{p}\right]$-module $k[X]$.

Nousiainen [N1] further gives the following example to show that $k[X]$ need not be a finitely generated $k[F]$-module (when $J(F)$ is invertible), thus negating a conjecture of Wang [Wa]: Take $n=2, F_{1}=X, F_{2}=Y+X Y^{p}$ (writing $X, Y$ for $X_{1}, X_{2}$ ). Then

$$
J(F)=\left(\begin{array}{cc}
1 & 0 \\
Y^{p} & 1
\end{array}\right)
$$

is invertible, $k[X, Y]=k\left[F_{1}, F_{2}\right][Y]$, and the equation of minimal degree of $Y$ over $k[F]$ is $F_{1} Y^{p}+Y-F_{2}=0$, so that $Y$ is not integral over $k[F]$.

Nousiainen in [N2] studies the invariant

$$
j(k)=\operatorname{Inf}\{[k(X): k(F)] \mid \operatorname{det} J(F)=1, k[X] \neq k[F]\},
$$

defined for any field $k$. The Jacobian Conjecture states that $j(k)=\infty$ if $\operatorname{char}(k)=0$. If $\operatorname{char}(k)=p$ we have $j(k) \leqslant p \quad\left(n=1, F=X+X^{p}\right)$ and $j(k)>1((\mathrm{a}) \Leftrightarrow(\mathrm{b})$ in (2.1)). Nousiainen shows that if $k$ is an ultraproduct, $k=\Pi_{थ} k_{\alpha}$ then $j(k)>j\left(k_{\alpha}\right)$ for "almost all" $\alpha$. With this he deduces for example that the Jacobian Conjecture follows if $\lim _{p} j\left(\mathbf{F}_{p}\right)=\infty$.

We now discuss the proof of (2.1). In the exact sequence,

$$
\Omega_{k[F] / k} \otimes_{k[F]} k[X] \stackrel{J}{\rightarrow} \Omega_{k[X] / k} \rightarrow \Omega_{k[X] / k[F]} \rightarrow 0
$$

the matrix of $J$ with respect to the bases $\left(d F_{i} \otimes 1\right)$ and $\left(d X_{i}\right)$, respectively, is $J(F)$. Thus

$$
\begin{aligned}
J(F) \text { invertible } & \Leftrightarrow \Omega_{k[X] / k[F]}=0 \\
& \Leftrightarrow k[X] / k[F] \text { is unramified. }
\end{aligned}
$$


If $x \in k^{n}$ and $y=F(x)$ the inclusion of local rings $k[F]_{y} \rightarrow k[X]_{x}$ is unramified and they have the same residue class field $k$, hence the same completions [A $+\mathbf{K}, \mathrm{VI},(3.7)]$. Consequently $F$ is étale at $x$ [A $+\mathbf{K}, \mathrm{VI},(4.5)]$. Passing to the algebraic closure of $k$ we see that $F$, being étale at all closed points, is étale [A + K, VI, (4.6)]. Thus

(2) $J(F)$ invertible $\Leftrightarrow k[X] / k[F]$ is étale (= flat and unramified).

Flatness has the following useful consequence.

$$
k[X] \cap k(F)=k[F] .
$$

In fact let $c=p / q \in k[X]$ where $p, q$ are relatively prime elements of $k[F]$. Then the homomorphism $k[F] / q k[F] \stackrel{p}{\rightarrow} k[F] / q k[F]$ is injective, so by the flat base change $k[X] \otimes_{k[F]^{-}}$, yields an injective homomorphism $k[X] / q k[X] \stackrel{p}{\rightarrow} k[X] / q k[X]$. This signifies that $p$ and $q$ are relatively prime in $k[X]$. But since $c=p / q \in k[X]$ we must then have $q \in k[X]^{\times}=k^{\times}$and so $c \in k[F]$.

Now to the proof of (2.1).

(a) $\Rightarrow$ (c): Trivial.

(c) $\Rightarrow($ b): In view of $(1), k(X) / k(F)$ is separable so a generic fibre of $F$ has $[k(X): k(F)]$ elements. Therefore when $F$ is injective, $[k(X): k(F)]=1$.

(b) $\Rightarrow$ (a): This is immediate from (3).

(a) $\Rightarrow$ (g): Trivial.

(a) $\Rightarrow$ (e): Trivial.

(e) $\Leftrightarrow\left(\mathrm{e}^{\prime}\right)$ : A theorem of Chevalley asserts the equivalence of properness and finiteness for any affine morphism (cf. [Mu, Lemma (3.5.1)]). Note that when $k=\mathbf{C}$ properness in the topological and algebraic-geometric senses coincide.

(e) $\Rightarrow$ (f): Use (2) and the fact that a flat module is projective if it is finitely presented, e.g. finitely generated over a noetherian ring.

(f) $\Rightarrow(e)$ : Over any integral domain a projective module of finite rank is finitely generated.

(e) $\Rightarrow$ (d): If $F$ is finite then $\overline{k[F]}=k[X]$, which is unramified over $k[F]$ by (1).

Suppose now that $k=\mathbf{C}$.

(d) $\Rightarrow$ (b): If $\overline{\mathbf{C}[F]} / \mathbf{C}[F]$ is unramified then (as in the derivation of (3) above), it is étale and finite, and so

$$
\operatorname{Spec}(\overline{\mathbf{C}[F]}) \rightarrow \operatorname{Spec}(\mathbf{C}[F]) \cong \mathbf{C}^{n}
$$

is a (connected) covering. Since $\mathbf{C}^{n}$ is simply connected we have $\overline{\mathbf{C}[F]}=\mathbf{C}[F]$, and hence $\mathbf{C}(F)=\mathbf{C}(X)$.

$(\mathrm{g}) \Rightarrow(\mathrm{b})$ : Consider the commutative diagram

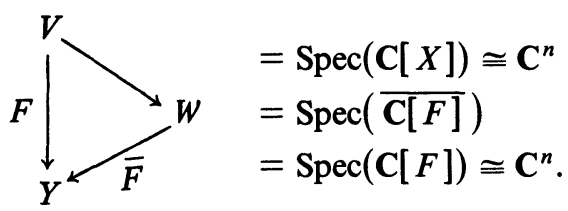


By Zariski's Main Theorem the birational morphism $V \rightarrow W$ of normal varieties is an open immersion; we identify $V$ with an open subvariety of $W$. It is contained in the set $U$ of points of $W$ where $\bar{F}$ is étale, since $F$ is étale. The group $\Gamma=\operatorname{Gal}(\mathbf{C}(X) / \mathbf{C}(F))$ acts freely on $U$ so that $\bar{F}: U \rightarrow \bar{F}(U)$ is a galois covering with group $\Gamma$. The morphisms $\bar{F}$ and $F$, being flat, are open $[\mathbf{A}+\mathbf{K}$, Chapter V, Theorem 5.1]. We have $\operatorname{Codim}_{Y}(Y-\bar{F}(U)) \geqslant \operatorname{Codim}_{Y}(Y-F(V))$ $\geqslant 2$ since $\mathbf{C}[F]$ is a UFD and $\mathbf{C}[X]^{\times}=\mathbf{C}^{\times}$. It follows that $\bar{F}(U)$ is simply connected, so the covering $U \rightarrow \bar{F}(U)$ is trivial. Therefore $\bar{F}$, and so also $F$, are birational.

Much of Theorem (2.1) is folklore. Many of the arguments above are adapted from Wang [Wa], especially the implications in positive characteristic. The most substantial result is $(\mathrm{g}) \Rightarrow(\mathrm{a})$. This was first proved by Campbell [C], who remarks several of the other equivalences as well. He uses methods of several complex variables. As remarked above Abhyankar [Ab1] proved the case $n=2$. Algebraic proofs of $(\mathrm{g}) \Rightarrow$ (a) can be found in Razar [R], Wright [Wr2], and Oda [O]. Wright gives an algebraic proof of the simple connectivity of $\mathbf{C}^{n}$, as well as the following differential criterion for the Jacobian Conjecture, discovered independently by $\mathbf{P}$. Nousiainen (cf. $[\mathbf{N}+\mathbf{S}])$.

Define derivations $\Delta_{i}: \mathrm{C}[X] \rightarrow \mathrm{C}[X]$ by

$$
\Delta_{i}(G)=\operatorname{det} J\left(F_{1}, \ldots, F_{i-1}, G, F_{i+1}, \ldots, F_{n}\right) .
$$

Assuming that $\operatorname{deg} J(F)=1$ we have

$$
\Delta_{i}\left(F_{j}\right)=\delta_{i j} \quad(i, j=1, \ldots, n) .
$$

Thus the $\Delta_{i}$ 's commute in $\mathbf{C}[F]$ and hence also in $\mathrm{C}[X]$ since $\Omega_{\mathrm{C}[X] / \mathrm{C}[F]}=0$ ((1) above). Since the $\partial / \partial F_{i}$ form a basis of $\Omega_{\mathrm{C}[F] / \mathrm{C}}$ it follows, again from (1), that $\Delta_{1}, \ldots, \Delta_{n}$ is a $\mathbf{C}[X]$-basis of $\Omega_{\mathbf{C}[X] / \mathbf{C}}$. In particular the $D_{i}$ are $\mathbf{C}[X]$-linear combinations of $\Delta_{1}, \ldots, \Delta_{n}$. It follows that $\bigcap_{i} \operatorname{Ker}\left(\Delta_{i}\right) \subset \bigcap_{i} \operatorname{Ker}\left(D_{i}\right)=\mathbf{C}$. Finally note that the $\Delta_{i}$ are locally nilpotent in $C[F]$.

(2.3) Proposition (CF. [Wr2, Proposition 2.2] or [N $+\mathbf{S}]$ ). If the derivations $\Delta_{i}$ are locally nilpotent (in $\mathbf{C}[X]$ ) then $\left.\mathbf{C}[F]=\mathbf{C} X\right]$.

The derivations $\Delta_{i}$ can be used to relate the Jacobian Conjecture to a problem about the Weyl algebra $A_{n}=\mathrm{C}\left[X_{1}, \ldots, X_{n}, D_{1}, \ldots, D_{n}\right]$ pointed out to us by L. Vaserstein and by V. Kac. Here we identify $A_{n}$ with an algebra of linear operators on $\mathrm{C}[X], X_{i}$ being identified with multiplication by $X_{i}$. Then we have the relations $\left[X_{i}, X_{j}\right]=0=\left[D_{i}, D_{j}\right]$ and $\left[D_{i}, X_{j}\right]=\delta_{i j}(1 \leqslant i, j \leqslant n)$. Moreover these relations define a presentation of $A_{n}$.

It is unknown whether every endomorphism of the C-algebra $A_{n}$ is an automorphism. If this were so the Jacobian Conjecture would follow. Indeed, given $F$ as above with det $J(F)=1$, define $\Delta_{i}$ as above; then we have an endomorphism $\varphi$ of $A_{n}$ defined by $\varphi\left(X_{i}\right)=F_{i}, \varphi\left(D_{i}\right)=\Delta_{i}(i=1, \ldots, n)$, since the defining relations are preserved. Suppose that $\varphi$ is an automorphism of $A_{n}$. Then since $\operatorname{ad}\left(D_{i}\right): H \mapsto\left[D_{i}, H\right]$ is a locally nilpotent derivation of $A_{n}$, it follows that $\operatorname{ad}\left(\Delta_{i}\right)$ is likewise locally nilpotent. It follows then from (2.3) that $\mathbf{C}[F]=\mathbf{C}[X]$, as claimed.

We mention finally the following significant result. 
(2.4) THeOREM (S. WANG [Wa]). Let $k$ be a field of characteristic $\neq 2$. Let $F \in \operatorname{End}\left(\mathbf{A}_{k}^{n}\right)$ have invertible Jacobian $J(F)$. Assume that $\operatorname{deg}(F) \leqslant 2$. Then $F$ is invertible.

This result was rediscovered by $\mathrm{S}$. Oda [O], with a much simpler proof than Wang's. A slight elaboration of Oda's argument is presented in [Wr2, Lemma 3.5]. The argument is so short that we reproduce it here. In view of Theorem (2.1), condition (c), it suffices to show that $F$ is injective. Suppose, on the contrary, that $F(a)=F(b)$ with $a \neq b$ in $k^{n}$. Replacing $F(X)$ by $G(X)=$ $F(X+a)-F(a)$, we have $0=G(0)=G(c)$ where $c=b-a \neq 0$, and $G$ is still quadratic; write $G=G_{(1)}+G_{(2)}$ where the components of $G_{(d)}$ are homogeneous of degree $d(d=1,2)$. Then

$$
\begin{aligned}
0 & =G(c)=G_{(1)}(c)+G_{(2)}(c) \\
& =G_{(1)}(c)+2 t_{0} G_{(2)}(c) \quad\left(t_{0}=1 / 2\right) \\
& =\left.\frac{d}{d t}\left(G_{(1)}(c) t+G_{(2)}(c) t^{2}\right)\right|_{t=t_{0}} \\
& =\left.\frac{d}{d t}(G(t c))\right|_{t=t_{0}}=J(G)\left(t_{0} c\right) \cdot c .
\end{aligned}
$$

Since $J(G)$, like $J(F)$, is invertible, and $c \neq 0$, this is a contradiction.

This theorem tempts one to conjecture, as does Oda, that the Jacobian Conjecture might be true in characteristic $p>0$ for morphisms $F$ of degree $d<p$. However this cannot be so since we show below that the Jacobian Conjecture (in any characteristic) follows once it is known for morphisms of degree $\leqslant 3$.

3. Faulty proofs. Several alleged proofs of the Jacobian Conjecture have been published. While it is "well known to the experts" that these proofs are faulty, it proved surprisingly difficult for us to document a precise critique, even unpublished, for each of them. It is therefore perhaps worthwhile to publish this account of our findings.

W. Engel [E] in 1955 claimed to prove the case $n=2$. Vitushkin [V] in 1975 published two essential errors in Engel's argument.

B. Segre has published three incomplete proofs of the Jacobian Conjecture. The first one in 1956 [Se1, \$22, no. 130] argues essentially as follows. Consider the geometric degree $d=[\mathbf{C}(X): \mathbf{C}(F)]$ of $F$. From Keller $[\mathbf{K}]$ one knows that $F$ is invertible in the birational case $(d=1)$, so one seeks to show that $d=1$. Modifying $F$ by an affine linear automorphism (Remark (1.1)1) we may assume that for each $i=1, \ldots, n$,

$$
F_{i}=X_{i}+F_{i(2)}+F_{i(3)}+\cdots
$$

with each $F_{i(r)}$ homogeneous of degree $r$. The invertibility of $J(F)$ then implies that det $J(F)=1$. Let $t \in \mathbf{C}^{\times}$and conjugate $F$ by the homothetic $X \mapsto t X$ to obtain $F^{t}$ with

$$
F_{i}^{t}=X_{i}+t F_{i(2)}+t^{2} F_{i(3)}+\cdots
$$


Note that $J\left(F^{t}\right)(X)=J(F)(t X)$ so that det $J\left(F^{t}\right)=1$. Moreover (1) defines $F^{t}$ even for $t=0$, when $F^{0}=X$ (the identity morphism). Thus (1) describes a homotopy from $F=F^{1}$ to the identity $F^{0}$. Evidently the geometric degree of $F^{t}$ is the same $d$ for all $t \in \mathbf{C}^{\times}$. Segre pretends to reason that this remains so for $t=0$, whence $d=1$. This argument is couched in terms of evaluation of a certain volume integral on $\mathbf{P}^{n}$ in which $t$ figures as a parameter, and the claim that the integral varies continuously with $t$.

Now it is known that the geometric degree is a homotopy invariant only for proper morphisms. If indeed Segre could verify that $F$ was proper then, as we saw in Theorem (2.1), the invertibility of $F$ would easily follow. Of course the $F^{t}$ above are proper outside a proper subvariety $V^{t}$, but $V^{t}$ varies with $t$ so the difficulty remains.

Segre's second attempt [Se2], shortly afterward, makes the stronger claim that if $\operatorname{det} J(F)=1$ then $F$ belongs to the group $E A_{n}(k)$ generated by "elementary" or "Jonquieres" automorphisms. ( $F$ is elementary if, for some $j$, $F_{i}=X_{i}$ for $i \neq j$ and $F_{j}-X_{j}$ is independent of $X_{j}$.) This claim is equivalent to the conjunction of the Jacobian Conjecture and the "Tame Generator Conjecture" that $G A_{n}(k)$ is generated by $E A_{n}(k)$ and $G L_{n}(k)$. Segre asserts that the claim is very likely a theorem for all $n$, and he offers a proof for $n=2$. He cites the earlier proof by Jung [Ju] of the Tame Generator Conjecture for $n=2$, and the "proof" by Engel [E] of the Jacobian Conjecture for $n=2$, as well as Segre's own earlier "proof" of the Jacobian Conjecture for general $n$ [Se1].

Segre rightly calls attention to the following lemma in his proof as having a special interest.

SEGRE'S LEMMA. If $f$ and $g$ in $\mathrm{C}[T]$ are nonconstant and generate the polynomial algebra $\mathrm{C}[T]$ then one of $\operatorname{deg}(f)$ and $\operatorname{deg}(g)$ divides the other.

Segre derives as a consequence of this that every embedding of the line in the plane is equivalent to a linear embedding.

Canals and Lluis $[\mathbf{C}+\mathbf{L}]$ later noted an error in Segre's proof of his lemma, and published a correction.

The fundamental paper of Abhyankar and Moh $[\mathbf{A}+\mathbf{M}]$, Embeddings of the line in the plane, states Segre's Lemma as its "Main Theorem," and takes is title from the above consequence. (Their version also applies in characteristic $p>0$.) It is pointed out that not only is Segre's proof faulty, but so also is the correction by Canals and Lluis, as conceded in correspondence quoted from the latter.

However there is strangely no reference to Segre's attempted proof of the Jacobian Conjecture, in which his lemma, now correctly proved by Abhyankar and Moh, figures. After considerable inquiry, Joel Roberts has kindly communicated to us the following critique of that aspect of Segre's paper.

Segre considers a map $F=(f, g): \mathbf{C}^{2} \rightarrow \mathbf{C}^{2}$ with $J(F)$ invertible. On p. 16 of [Se2] he chooses $(a, b) \in \mathbf{C}^{2}$ where the leading homogeneous terms of $f$ and $g$ are nonzero, and he introduces the map $P: C \rightarrow C^{2}$ defined by $P(t)=$ $F(t a, t b)$. From the Chain Rule and invertibility of $J(F)$ one sees that $(d / d t) f(t a, t b)$ and $(d / d t) g(t a, t b)$ have no common zeros, and hence that $P$ 
is an immersion. However Segre claims [Se2, p. 16, lines 7-9] that the curve $P(\mathbf{C}) \subset \mathbf{C}^{2}$ is nonsingular, which is equivalent to $P$ being injective. This of course must be so if $F$ is to be invertible, but no adequate justification of the claim is given by Segre.

In 1960 Segre proposed a third proof of the Jacobian Conjecture [Se3, §14], couched in topological terms. He views $F: \mathbf{C}^{n} \rightarrow \mathbf{C}^{n}$ as a rational map from $V=\mathbf{P}^{n}$ to $V^{\prime}=\mathbf{P}^{n}$ defined and locally invertible outside the hyper-plane $H_{\infty}$ at $\infty$. He takes from $V^{\prime}$ the subvariety $W^{\prime}$ consisting of the branch locus and all points with positive dimensional $F$-fiber. Then he asserts that $F$ defines a (topological) covering from $V^{*}=V-H_{\infty}\left(=\mathbf{C}^{n}\right)$ to $V^{\prime *}=V^{\prime}-W^{\prime}$. In fact it is not clear why $F$ even maps $V^{*}$ into $V^{\prime *}$, and, if so, why, in the absence of properness conditions on $F$, such a map must be a covering.

Segre in [Se3] proposed the problem of finding a purely algebraic proof of the Jacobian Conjecture. Gröbner responded with such a proof in 1961 [Gr]. Zariski pointed out to Barsotti, reviewer of the article, that "formula (14) on p. 121 contains a computational error which invalidates the proof." Indeed, Marilena Pittaluga has shown us an automorphism of $\mathbf{C}^{2}$ for which the derivatives $\partial x_{1}^{\prime} / \partial y_{j}^{\prime}$ Gröbner claims, using (14), are finite, are in fact infinite. She notes, moreover, that in deriving a contradiction Gröbner never uses his hypothesis that his map is not birational, so that the argument has to be faulty.

Shafarevic [Sh] in 1967 gave the group $G A_{n}(\mathbf{C})$ as an example of an infinite dimensional algebraic group (in the sense he there introduced), stating that it was defined in the space of polynomial $n$-tuples $F=\left(F_{1}, \ldots, F_{n}\right)$ by the condition det $J(F) \in \mathbf{C}^{\times}$. His belief that this had been established was apparently an oversight, as he later conceded in private communications. In this connection Corollary (1.6) is relevant.

S. Oda's preprint [O] contains an alleged proof of the Jacobian Conjecture (Theorem 4 of [O]). The error in Oda's argument occurs in his Lemma 2(e), which is misquoted from Murre ([Mu, p. 39]), and which is trivially false in the form stated by Oda.

4. The use of stabilization and formal methods. We present below what appears to be a new approach to the Jacobian Conjecture, based on two simple ideas.

The first is the "stabilization philosophy" of algebraic $K$-theory: A problem posed in dimension $n$ may become simpler if looked at in dimension $n+m$. This is implemented here as follows:

Let $F=\left(F_{1}, \ldots, F_{n}\right) \in \operatorname{End}\left(\mathbf{A}_{k}^{n}\right)$ have invertible Jacobian $J(F)$. For any $m \geqslant 0$ consider

$$
F^{[m]}=\left(F_{1}, \ldots, F_{n}, X_{n+1}, \ldots, X_{n+m}\right) \in \operatorname{End}\left(\mathbf{A}_{k}^{n+m}\right) .
$$

Then

$$
J\left(F^{[m]}\right)=\left(\begin{array}{cc}
J(F) & 0 \\
0 & I_{m}
\end{array}\right)
$$

is still invertible, and $F$ is invertible iff $F^{[m]}$ is invertible. Now we are able to show that for $m$ large, there exist $G, H \in G A_{n+m}(k)$ such that $E=$ $G \circ F^{[m]} \circ H$ is a specialization of a morphism of the form $X+N$ where each 
$N_{i}$ is a cubic homogeneous polynomial and $J(N)$ is nilpotent (Theorem (2.1) of Chapter II). ${ }^{5}$

The other idea is embodied in Remark (1.1)2 above: assuming that $F(0)=0$ and $J(F)(0)$ is invertible then $F$ has a formal inverse $G=\left(G_{1}, \ldots, G_{n}\right)$. Thus the Jacobian Problem is not whether an inverse exists, but rather whether this formal inverse $G$ is really a polynomial when $J(F)$ is invertible. To seriously consider the problem from this point of view one needs a formula for $G$. Until recently such inversion formulas were relatively inaccessible, which presumably explains why this quite natural approach has not earlier been pursued.

The inversion formulas in question are of the following type. Let $F=$ $\left(F_{1}, \ldots, F_{n}\right)$ where for each $i, F_{i} \in k\left[\left[X_{1}, \ldots, X_{n}\right]\right]=k^{[[n]]}$ and $F_{i}=X_{i}+$ terms of degree $\geqslant 2$. We seek formulas for the unique $G_{1}, \ldots, G_{n}$ in $k^{[[n]]}$ such that $G_{i}(F)=X_{i}(i=1, \ldots, n)$. In the classical case one has $k=\mathbf{C}$. The case $n=1$ goes back to Lagrange. Call $F$ "diagonal" if $F_{i}=X_{i} H_{i}$ for some $H_{i} \in k^{[[n]]}$; this is automatic if $n=1$. Lagrange's inversion was generalized to $n$ variables in the diagonal case by I. J. Good [G] in 1960. Good cites earlier treatments of the case $n=2$ by Stieltjes and Poincaré, and independently, by Goursat. Ira Gessel recently pointed out to us that, in fact, Good's inversion formula was published (in Latin) already by Jacobi in 1830 [J].

To the best of our knowledge the first general inversion formula was derived by Gurjar (unpublished) in 1974, using Goursat's complex variable methods. Gurjar was then a student of Abhyankar, who found a simpler form and algebraic proof of the inversion formula. This appears in Abhyankar's Purdue Lecture notes [Ab2] in 1974. Since it is so fundamental for the sequel, we reproduce Abhyankar's formula and proof here in Chapter III (Theorem (2.1)).

Since writing the first draft of this paper we discovered first Good's paper [G], covering the diagonal case, and later the 1978 paper of S. A. Joni [Jo]. Joni, in her 1976 dissertation, apparently rediscovered a general inversion formula without knowledge of Abhyankar's.

For application to the Jacobian Conjecture we assume, thanks to the results of Chapter II, that $F_{i}=X_{i}-H_{i}$ where $H$ is a cubic homogeneous form and $J(G)$ is nilpotent. The final result is an expansion of the inverse series $G_{i}$ indexed by finite, rooted, and labeled trees (Chapter III, Theorem (4.1)). This formula gives some hope of showing that $G_{i}$ is a polynomial. The method it affords is illustrated by calculations in Chapter III, $\$ 5$, where a precise Conjecture (5.1) is formulated.

It is remarkable that Good [G] also uses his inversion formula for problems of enumerating rooted colored trees. This suggests perhaps some combinatorial interest of our formula.

\footnotetext{
${ }^{5}$ Since writing this paper L. Avramov has kindly brought to our attention the very interesting paper of Jagžev [Ja] (1980). Jagžev proves a theorem substantially equivalent to our Reduction Theorem (2.1) of Chapter II, though with some differences of detail, and by somewhat different methods. He rediscovers the implication (c) $\Rightarrow$ (a) of Theorem (2.1) above, and uses it, as in [Wr2], to prove Wang's Theorem ((2.4) above), which, he, like Oda [O], rediscovered. He further uses it to show that, for $F=X+N$ as above, $F$ is invertible provided that $J(F)(X)+J(F)(Y)$ is invertible over $k^{[2 n]}$, where $Y=\left(Y_{1}, \ldots, Y_{n}\right)$ denotes $n$ new indeterminates.
} 
The discovery of Abhyankar's inversion formula gave the original impetus for this work, which may fairly be considered to be an effort in High School Algebra, in Abhyankar's sense [Ab3].

We are grateful to numerous colleagues for helpful comments on this work, notably to the auditors of Bass' lectures on it at the Tata Institute in the fall of 1980, especially R. Raghunathan. Specifically we are indebted to Joel Roberts for a critique of Segre's paper [Se2], to Francesco Scattone for translation of [Se3], to Marilena Pittaluga for a translation and critique of [Gr], to Ofer Gabber for Theorem (1.5) of Chapter I and for some simplifications of Chapter III, to M. P. Murthy for collaboration on Theorem (6.2) of Chapter II, which proves the Jacobian Conjecture for $F=X-H$ when $J(H)$ is nilpotent of rank 1, to L. Avramov for help in translating the new paper of Jagzev [Ja], and to Dan Grayson for computer tests of our Conjecture (5.1) (which implies the Jacobian Conjecture). Finally we thank Kate March and Clara Gama for typing of the manuscript, and Dorothea Goldys-Bass for the tree figures in $\$ 5$ of Chapter III.

\section{The REDUCTION THEOREM; DEgREE 3 WITH UNIPOTENT JACOBIAN}

1. Notation. $k$ denotes a commutative ring. For each integer $n \geqslant 0$ we put

$$
\mathbf{n}=\{1,2, \ldots, n\}, \text { and } X_{\mathbf{n}}=\left(X_{1}, \ldots, X_{n}\right)
$$

where the $X_{i}$ are indeterminants. When $n$ is understood we often write $X$ for $X_{n}$. We have the polynomial algebra,

$$
k^{[n]}=k[X]=k\left[X_{1}, \ldots, X_{n}\right],
$$

and we put

$$
M A_{n}(k)=\left(k^{[n]}\right)^{n}=\left\{F=\left(F_{1}, \ldots, F_{n}\right) \mid F_{i} \in k^{[n]}, \quad i=1, \ldots, n\right\} .
$$

On $M A_{n}(k)$ we consider the following two structures.

1. Monoid. The composition is

$$
F \circ G=F(G)=\left(F_{1}(G), \ldots, F_{n}(G)\right),
$$

with neutral element $X$. This monoid is isomorphic to $\operatorname{End}\left(\mathbf{A}_{k}^{n}\right)$, and antiisomorphic to $\operatorname{End}_{k-\text { alg }}\left(k^{[n]}\right)$. Its group of invertible elements is the affine Cremona group

$$
G A_{n}(k) \cong \operatorname{Aut}\left(\mathbf{A}_{k}^{n}\right) .
$$

2. Graded $k$-algebra. We give $M A_{n}(k)$ the (cartesian) product ring structure (coordinatewise operations) with grading

$$
M A_{n}(k)_{(d)}=\left(k_{d}^{[n]}\right)^{n}
$$

coming from the grading of $k^{[n]}$ by the modules $k_{d}^{[n]}$ of forms in $X$ of degree $d$ for each $d \geqslant 0$. If $F \in M A_{n}(k)$ we write $F=\Sigma_{d \geqslant 0} F_{(d)}$ where $F_{(d)}=$ $\left(F_{(d), 1}, \ldots, F_{(d), n}\right)$ is the $d$ th homogeneous component of $F$, and $F_{(0)}=F(0)$. The largest $d$ for which $F_{(d)} \neq 0$ is called the degree of $F$, and denoted $\operatorname{deg}(F)$. 
We have the Jacobian

$$
\begin{aligned}
J: M A_{n}(k) & \rightarrow M_{n}\left(k^{[n]}\right), \\
J(F) & =\left(\begin{array}{ccc}
D_{1} F_{1} & \cdots & D_{n} F_{1} \\
\vdots & & \vdots \\
D_{1} F_{n} & \cdots & D_{n} F_{n}
\end{array}\right) \quad\left(D_{i}=\frac{\partial}{\partial X_{i}}\right),
\end{aligned}
$$

which satisfies

$$
J(G(F))=J(G)(F) \cdot J(F) .
$$

Note that $J(F)(0)=J\left(F_{(1)}\right)(0)$ is just the matrix corresponding to the linear endomorphism $F_{(1)}$ of $\mathbf{A}_{k}^{n}$.

We put

$$
\begin{aligned}
M A_{n}^{0}(k) & =\left\{F \in M A_{n}(k) \mid F(0)=0\right\} \\
& =\bigoplus_{d>1} M A_{n}(k)_{(d)} .
\end{aligned}
$$

This is an ideal of the ring $M A_{n}(k)$, and

$$
\begin{aligned}
J_{0}: M A_{n}^{0}(k) & \rightarrow M_{n}(k), \\
J_{0}(F) & =J(F)(0)
\end{aligned}
$$

is a monoid homomorphism, whose kernel we denote

$$
\begin{aligned}
M A_{n}^{1}(k) & =\{F \mid F(0)=0 \text { and } J(F)(0)=I\} \\
& =\left\{F \mid F \equiv X \bmod M A_{n}^{0}(k)^{2}\right\} .
\end{aligned}
$$

For any $d \geqslant 0$ we could thus consistently define

$$
M A_{n}^{d}(k)=\left\{F \mid F \equiv X \bmod M A_{n}^{0}(k)^{d+1}\right\} .
$$

This corresponds to the kernel of the natural homomorphism

$$
\operatorname{End}_{k-\mathrm{alg}}^{0}\left(k^{[n]}\right) \rightarrow \operatorname{End}_{k-\mathrm{alg}}\left(k^{[n]} /(X)^{d+1}\right),
$$

where the superscript 0 denotes endomorphisms stabilizing the ideal $(X)$ of $k^{[n]}$. We put

$$
G A_{n}^{d}(k)=G A_{n}(k) \cap M A_{n}^{d}(k) \quad(d \geqslant 0) .
$$

(1.1) Proposition. Let $F \in M A_{n}(k)$. Then

$$
F=(X+F(0)) \circ F_{+}
$$

where $F_{+}=F_{(1)}+F_{(2)}+\cdots \in M A_{n}^{0}(k)$. If $J(F)(0)$ (or, equivalently, $\left.F_{(1)}\right)$ is invertible then

$$
F=(X+F(0)) \circ F_{(1)} \circ F^{\prime}
$$

where $F^{\prime} \in M A_{n}^{1}(k)$. Moreover $F$ is invertible iff $F^{\prime}$ is invertible. 
This is clear, and it shows that, for the Jacobian Conjecture, one may restrict attention to elements $F \in M A_{n}^{1}(k)$. For $G A_{n}(k)$, (1) gives a decomposition into the product,

$$
G A_{n}(k)=T_{n}(k) \cdot G L_{n}(k) \cdot G A_{n}^{1}(k),
$$

where $T_{n}(k)$ is the group of translations and $G L_{n}(k)$ is identified with the group of linear homogeneous elements of $G A_{n}(k)$.

Call $F \in M A_{n}(k)$ elementary if, for some $j, F_{i}-X_{i}$ is zero for $i \neq j$, and independent of $X_{j}$ for $i=j$. Such an $F$ is clearly an automorphism $\left(\left(F^{-1}\right)_{i}-X_{i}\right.$ $\left.=-\left(F_{i}-X_{i}\right)\right)$. The group generated by all elementary automorphisms is denoted $E A_{n}(k)$, and we put

$$
E A_{n}^{d}(k)=E A_{n}(k) \cap M A_{n}^{d}(k) \quad(d \geqslant 0) .
$$

For $m \geqslant 0$ we define the stabilization map

$$
\begin{aligned}
M A_{n}(k) & \rightarrow M A_{n+m}(k), \\
F & \mapsto F^{[m]},
\end{aligned}
$$

where

$$
F^{[m]}=\left(F_{1}, \ldots, F_{n}, X_{n+1}, \ldots, X_{n+m}\right) .
$$

This is a monomorphism of monoids. Observe that

$$
J\left(F^{[m]}\right)=\left(\begin{array}{cc}
J(F) & 0 \\
0 & I_{m}
\end{array}\right)
$$

and that $F$ is invertible iff $F^{[m]}$ is invertible. Further $M A_{n}^{d}(k)^{[m]} \subset$ $M A_{n+m}^{d}(k)$ for $d \geqslant 0$ and $E A_{n}(k)^{[m]} \subset E A_{n+m}(k)$. Passing to the limit as $n \rightarrow \infty$ one obtains $E A(k) \subset G A(k)$. Conjecturally the commutator subgroup $[G A(k), G A(k)]$ equals $E A(k)$. Connell [Co] has shown that it is at least the normal closure of $E A(k)$.

\section{Statement of the Reduction Theorem.}

(2.1) THEOREM. Let $k$ be a commutative ring, and let $F \in M A_{n}^{1}(k)$ have invertible Jacobian $J(F)$. There exist an integer $m \geqslant 0$, elements $G, H \in$ $E A_{n+m}^{0}(k)$, and $\tilde{F}(T) \in M A_{n+m}(k[T])$, where $T$ is an indeterminate, with the following properties.

(a) For $T=1$ we have $\tilde{F}(1)=G \circ F^{[m]} \circ H$. Thus if $\tilde{F}$ is invertible then so also is $F$.

(b) The $k[T]$-algebra endomorphism $\varphi_{\tilde{F}}$ of $k[T]^{[n+m]}$ defined by $\tilde{F}$ can be viewed as a $k$-algebra endomorphism of $k\left[X_{1}, \ldots, X_{n+m}, T\right]=k^{[r]}$, where $r=n$ $+m+1$. As such it defines an element $L \in M A_{r}(k)$, which is invertible iff $\tilde{F}$ is. We have $L=X_{\mathrm{r}}+N$ where $N$ is cubic homogeneous $\left(N=N_{(3)}\right)$, and linear in each variable except quadratic in $T$, and $J(N)$ is nilpotent.

(2.2) Corollary. Suppose, for all $n$ and all $F \in M A_{n}(k)$ of the form $F=X+N$ with $N$ cubic homogeneous and $J(N)$ nilpotent, that $F$ is invertible. Then for all $n$ and all $F \in M A_{n}(k)$ with $J(F)$ invertible, $F$ is invertible. 
The Reduction Theorem will be proved in three steps, each of which furnishes slightly more general information.

Step 1. Reduction to degree 3 (\$3).

Step 2. Making $J(F)$ unipotent (\$4).

Step 3. Homogenization (\$4).

\section{Reduction to degree 3.}

(3.1) Proposition. Let $F \in M A_{n}(k)$. There is an integer $m \geqslant 0$ and elements $G, H$ in $E A_{n+m}^{1}(k)$ such that $F^{\prime}=G \circ F^{[m]} \circ H$ has degree $\leqslant 3$. By allowing $H$ to be taken from $E A_{n+m}^{0}(k)$ we can further arrange that $F^{\prime}$ be linear in each variable.

Put $d=\operatorname{deg}(F)$ and let $e$ denote the number of monomials of degree $d$ occurring in $F=\left(F_{1}, \ldots, F_{n}\right)$. We prove the first assertion by induction on $(d, e)$. If $d \leqslant 3$ there is nothing to prove, so assume that $d \geqslant 4$. Let $M$ be a monomial of degree $d$ occurring in $F$, say in $F_{1}$, with coefficient $a \in k$. Write $a M=P Q$ with $P$ and $Q$ both of degree $\leqslant d-2$. Consider the following elements of $E A_{n+2}^{1}(k)$.

$$
\begin{aligned}
& G=\left(X_{1}-X_{n+1} X_{n+2}, X_{2}, \ldots, X_{n}, X_{n+1}, X_{n+2}\right), \\
& H=\left(X_{1}, \ldots, X_{n}, X_{n+1}+P, X_{n+2}+Q\right) .
\end{aligned}
$$

Then $F^{\prime}=G \circ F^{[2]} \circ H$ is given by,

$$
F^{\prime}=\left(F_{1}^{\prime}, F_{2}, \ldots, F_{n}, X_{n+1}+P, X_{n+2}+Q\right),
$$

where

$$
\begin{aligned}
F_{1}^{\prime} & =F_{1}-\left(X_{n+1}+P\right)\left(X_{n+2}+Q\right) \\
& =\left(F_{1}-a M\right)-X_{n+1} Q-P X_{n+2}-X_{n+1} X_{n+2} .
\end{aligned}
$$

Clearly either $\operatorname{deg}\left(F^{\prime}\right)<d$ or else $\operatorname{deg}\left(F^{\prime}\right)=d$ and $e\left(F^{\prime}\right)<e$. By induction therefore there exist $m \geqslant 0$ and $G^{\prime}, H^{\prime} \in E A_{n+2+m}^{1}(k)$ such that $F^{\prime \prime}=$ $G^{\prime} \circ F^{[m]} \circ H^{\prime}$ has degree $\leqslant 3$. Since $F^{\prime \prime}=\left(G^{\prime} \circ G^{[m]}\right) \circ F^{[2+m]} \circ\left(H^{[m]} \circ H^{\prime}\right)$ and the outside factors belong to $E A_{n+2+m}^{1}(k)$, the first assertion is proved.

To prove the final assertion we may therefore assume that $F$ already has degree $\leqslant 3$. If $M=X_{1}^{e_{1}} \cdots X_{n}^{e_{n}}$ put $e_{j}(M)=e_{j}$. If $f \in k^{[n]}$ write $M \in f$ if $M$ occurs (with nonzero coefficient) in $f$, and put

$$
e(f)=\sum_{M \in f} \sum_{j=1}^{n} \operatorname{Max}\left(e_{j}(M)-1,0\right)^{2}
$$

Thus $e(f) \geqslant 0$ and $e(f)=0$ iff $f$ is linear in each variable. Put $e(F)=e\left(F_{1}\right)$ $+\cdots+e\left(F_{n}\right)$; we argue by induction on $e(F)$ to make $e(F)=0$.

Suppose that $e(F)>0$. Some $F_{i}$, say $F_{1}$, contains a monomial $M$, say with coefficient $a \in k$, divisible by $X_{j}^{2}$ for some $j$. Write $a M=P Q$ so that $X_{j}$ divides both $P$ and $Q$. Now define $G, H$, and $F^{\prime}=G \circ F^{[2]} \circ H$ as in formulas (1) and (2) above. Then $G \in E A_{n+2}^{1}(k), H \in E A_{n+2}^{0}(k)$, and one can verify from (2) and (3) that $e\left(F^{\prime}\right)<e(F)$, using the fact that $M=X_{j}^{2} N$ with $N$ of degree $\leqslant 1$. Now arguing as in the first part of the proof, we conclude by applying the induction hypothesis to $F^{\prime}$. 
4. Proof of the Reduction Theorem. Let $F \in M A_{n}^{1}(k)$ have invertible Jacobian $J(F)$. Let $F^{\prime}=G \circ F^{[m]} \circ H$ as in Proposition (3.1). Then $F^{\prime}=F_{(1)}^{\prime}+F_{(2)}^{\prime}+$ $F_{(3)}^{\prime}$ and $F^{\prime}$ is linear in each variable. Moreover

$$
F_{(1)}^{\prime}=G_{(1)} \circ F_{(1)}^{[m]} \circ H_{(1)}=H_{(1)} \in E A_{n+m}^{0}(k) .
$$

Consider

$$
F^{\prime \prime}=F_{(1)}^{\prime-1} \circ F^{\prime}=X+F_{(1)}^{\prime-1}\left(F_{(2)}^{\prime}\right)+F_{(1)}^{\prime-1}\left(F_{(3)}^{\prime}\right)
$$

in $M A_{n+m}^{1}(k)$. Clearly $F^{\prime \prime}$ is still linear in each variable (which is not necessarily the case for $\left.F^{\prime} \circ F_{(1)}^{\prime-1}\right)$, and $\operatorname{deg}\left(F^{\prime \prime}\right) \leqslant 3$. Replacing $F$ by $F^{\prime \prime}$ we are thus reduced to the case: $\operatorname{deg}(F) \leqslant 3$ and $F$ is linear in each variable.

Let $T$ be an indeterminate. Put

$$
E(T)=X+T F_{(2)}+T^{2} F_{(3)} \in M A_{n}^{1}(k[T]) .
$$

We have $J(E(T))=I+T J\left(F_{(2)}\right)+T^{2} J\left(F_{(3)}\right)=J(F)(T X)$, so that $J(E(T))$, like $J(F)$, is invertible. In $k^{[2 n]}$ denote the variables by $(X, Y)=$ $\left(X_{1}, \ldots, X_{n}, Y_{1}, \ldots, Y_{n}\right)$. Consider the elements

$$
\left.\begin{array}{l}
G(T)=(X+T Y, Y) \\
H(T)=\left(X, Y-T F_{(3)}\right)
\end{array}\right\} \in E A_{2 n}^{0}(k[T])
$$

and put $E^{\prime}(T)=G(T) \circ E(T)^{[n]} \circ H(T)$. We have

$$
\begin{aligned}
E^{\prime}(T) & =\left(E(T)+\left(Y-F_{(3)} T\right) T, Y-F_{(3)} T\right) \\
& =\left(\left(E(T)-F_{(3)} T^{2}\right)+Y T, Y-F_{(3)} T\right)=(X, Y)+N T
\end{aligned}
$$

where $N=\left(F_{(2)}+Y,-F_{(3)}\right)$. We have $J\left(E^{\prime}(T)\right)=I+J(N) T \in$ $G L_{2 n}\left(k^{[2 n]}[T]\right)$, and

$$
J(N)=\left(\begin{array}{cc}
J\left(F_{(2)}\right) & I \\
-J\left(F_{(3)}\right) & 0
\end{array}\right) .
$$

The following lemma applied to the ring $A=M_{2 n}\left(k^{[2 n]}\right)[T]$, graded by powers of $T$, and to the element $a=J(N) T \in A_{1}$, shows now that $J(N)$ is nilpotent.

(4.1) LemMA. Let $A=A_{0} \oplus A_{1} \oplus \cdots$ be a graded ring, and let $a \in A_{d}$ for some $d \geqslant 1$. Then $1+a$ is invertible iff $a$ is nilpotent.

In fact the inverse must be $\Sigma_{m>0}(-a)^{m}$.

Now setting $T=1$ we obtain the element

$$
\begin{aligned}
F^{\prime} & =E^{\prime}(1)=G(1) \circ E(1)^{[n]} \circ H(1) \\
& =G(1) \circ F^{[n]} \circ H(1)=(X, Y)+N
\end{aligned}
$$

where $G(1), H(1) \in E A_{2 n}^{0}(k)$ and $N=\left(F_{(2)}+Y,-F_{(3)}\right)$. Thus we may replace $F$ by $F^{\prime}$ and reduce to the case:

$F=X+N$,

$N=N_{(1)}+N_{(2)}+N_{(3)}$ is cubic and linear in each variable, and $J(N)$ is nilpotent. 
In this case we put

$$
\tilde{F}(T)=X+\tilde{N}(T), \quad \text { where } \tilde{N}(T)=N_{(1)} T^{2}+N_{(2)} T+N_{(3)} .
$$

For $T=1$ we have $\tilde{F}(1)=F$. Identifying $k^{[n+1]}$ with $k\left[X_{1}, \ldots, X_{n}, T\right]$, we have the $k$-endomorphism defined by $\tilde{F}$,

$$
L=\left(\tilde{F}_{1}, \ldots, \tilde{F}_{n}, T\right)=(\tilde{F}, T)=(X, T)+(\tilde{N}, 0)
$$

with Jacobian

$$
J(L)=I+\left(\begin{array}{c|c}
J_{X}(\tilde{N}) & { }^{t} N_{(2)}+2^{t} N_{(1)} T \\
\hline 0 & 0
\end{array}\right)
$$

which is unipotent iff $J_{X}(\tilde{N})$ is nilpotent. Since $\tilde{N}$ is cubic homogeneous in $(X, T)$, and linear in each $X_{i}$ and quadratic in $T$, we will have established all claims of the theorem once we show that $J_{X}(\tilde{N})$ is nilpotent.

Let $A=A_{0} \oplus A_{1} \oplus \cdots$ be a graded ring. Grade $A[T]$ by

$$
A[T]_{d}=A_{0} T^{d} \oplus A_{1} T^{d-1} \oplus \cdots \oplus A_{d} .
$$

Putting $T=1$ gives a linear isomorphism

$$
A[T] \stackrel{T=1}{\longrightarrow} A_{(d)}=A_{0} \oplus A_{1} \oplus \cdots \oplus A_{d} .
$$

Its inverse sends $a=a_{0}+a_{1}+\cdots+a_{d} \in A_{(d)}$ to $a^{(d)}=a_{0} T^{d}+a_{1} T^{d-1}$ $+\cdots+a_{d}$. Let $b \in A_{(e)}$ and put $c(T)=a^{(d)}(T) b^{(e)}(T) \in A[T]_{d+e}$. Since $c(1)=a^{(d)}(1) b^{(e)}(1)=a b \in A_{(d+e)}$ we conclude that

$$
a^{(d)} b^{(e)}=(a b)^{(d+e)} \text {. }
$$

Consequently

$$
\left(a^{(d)}\right)^{N}=\left(a^{N}\right)^{(N d)}
$$

for $N \geqslant 1$. It follows that $a$ is nilpotent iff $a^{(d)}$ is nilpotent.

Now take $A$ to be the ring $M_{n}\left(k^{[n]}\right)=M_{n}(k)^{[n]}$, graded by $X$-degree, and let $a=J(N)=J\left(N_{(1)}\right)+J\left(N_{(2)}\right)+J\left(N_{(3)}\right) \in A_{(2)}$. We have $a^{(2)}=J\left(N_{(1)}\right) T^{2}+$ $J\left(N_{(2)}\right) T+J\left(N_{(3)}\right)=J_{X}(\tilde{N})$. Since $J(N)$ is nilpotent it follows, as claimed, that $J_{X}(N)$ is nilpotent. This completes the proof of Theorem (2.1).

5. $T$-linearization and unipotent reduction. The argument in proving Theorem (2.1) to make $J(F)$ unipotent was applied only after $F$ was made cubic. We record here the information that argument yields in general.

(5.1) Proposition. Let $F \in M A_{n}(k[T])$ and write $F=F^{(0)}+F^{(1)} T$ $+\cdots+F^{(d)} T^{d}$ with each $F^{(j)} \in M A_{n}(k)$. Suppose that $d \geqslant 2$. There exist $G(T), H(T) \in E A_{d n}^{0}(k[T])$ such that $G(0)=H(0)=X_{\mathrm{dn}}$, and such that the element $F^{\prime}=G \circ F^{[(d-1) n]} \circ H$ has the following form: Denote the variables $X_{\mathrm{dn}}$ by $\left(X_{\mathrm{n}}^{(1)}, \ldots, X_{\mathrm{n}}^{(d)}\right)$ where $X_{\mathrm{n}}^{(j)}=\left(X_{1}^{(j)}, \ldots, X_{n}^{(j)}\right)(j=1, \ldots, d)$. Then

$$
\begin{aligned}
F^{\prime}= & \left(F^{(0)}, X_{\mathrm{n}}^{(2)}, \ldots, X_{\mathrm{n}}^{(d)}\right) \\
& +\left(F^{(1)}-X_{\mathrm{n}}^{(d)}, F^{(d)}, F^{(d-1)}-X_{\mathrm{n}}^{(2)}, \ldots, F^{(2)}-X_{\mathrm{n}}^{(d-1)}\right) T .
\end{aligned}
$$

Thus $F^{\prime}$ is linear in $T$, and involves the same $X$-monomials as $F^{[(d-1) n]}$. 
In the proof we shall write $X^{(j)}$ for $X_{n}^{(j)}$. We start with

$$
\begin{aligned}
& G_{1}=\left(X^{(1)}-X^{(2)} T^{d-1}, X^{(2)}\right), \\
& H_{1}=\left(X^{(1)}, X^{(2)}+F^{(d)} T\right)
\end{aligned}
$$

in $E A_{2 n}^{0}(k[T])$, and put $F_{1}=G_{1} \circ F^{[n]} \circ H_{1}$. (This abusive notation should not confuse $F_{1}$ with the first component function of $F$.) We have

$$
F_{1}=\left(\left(F-F^{(d)} T^{d}\right)-X^{(2)} T^{d-1}, X^{(2)}+F^{(d)} T\right) .
$$

Next take

$$
\begin{aligned}
& G_{2}=\left(X^{(1)}-X^{(3)} T^{d-2}, X^{(2)}, X^{(3)}\right), \\
& H_{2}=\left(X^{(1)}, X^{(2)}, X^{(3)}+\left(F^{(d-1)}-X^{(2)}\right) T\right)
\end{aligned}
$$

in $E A_{3 n}^{0}(k[T])$, and put $F_{2}=G_{2} \circ F_{1}^{[n]} \circ H_{2}=\left(F_{2}^{(1)}, F_{2}^{(2)}, F_{2}^{(3)}\right)$, where

$$
\begin{aligned}
& F_{2}^{(1)}=\left(F-F^{(d)} T^{d}-F^{(d-1)} T^{d-1}\right)-X^{(3)} T^{d-2}, \\
& F_{2}^{(2)}=X^{(2)}+F^{(d)} T, \\
& F_{2}^{(3)}=X^{(3)}+\left(F^{(d-1)}-X^{(2)}\right) T .
\end{aligned}
$$

Now we continue inductively to produce $F_{j}=G_{j} \circ F_{j-1}^{[n]} \circ H_{j}$, where

$$
\begin{array}{r}
G_{j}=\left(X^{(1)}-X^{(j+1)} T^{d-j}, X^{(2)}, \ldots, X^{(j+1)}\right), \\
H_{j}=\left(X^{(1)}, \ldots, X^{(j)}, X^{(j+1)}+\left(F^{(d-j+1)}-X^{(j)}\right) T\right),
\end{array}
$$

for $j=2, \ldots, d-1$. The $F^{\prime}$ of the proposition is $F_{d-1}$, which is easily seen to be of the alleged form.

(5.2) Proposition. Let $F \in M A_{n}^{1}(k)$ be of degree $\leqslant d+1$. There exist elements $G, H \in E A_{\mathrm{dn}}^{0}(k)$ such that the element $F^{\prime}=G \circ F^{[(d-1) n]} \circ H$ has the following form. Writing $X_{\mathrm{dn}}=\left(X_{\mathrm{n}}^{(1)}, \ldots, X_{\mathrm{n}}^{(d)}\right)$ as in Proposition (5.1), we have $F^{\prime}=X_{\mathrm{dn}}+N$ where

$$
N=\left(F_{(2)}-X_{n}^{(d)}, F_{(d+1)}, F_{(d)}-X_{n}^{(2)}, \ldots, F_{(3)}-X_{n}^{(d-n)}\right) .
$$

If $J(F)$ is invertible then $J(N)$ is nilpotent.

Put

$$
\begin{aligned}
E(T) & =\sum_{j=1}^{d+1} F_{(j)} T^{j-1} \\
& =X_{\mathbf{n}}+F_{(2)} T+\cdots+F_{(d+1)} T^{d} \in M A_{n}^{1}(d[T]) .
\end{aligned}
$$

Apply Proposition (5.1) to $E=\Sigma_{j=0}^{d} E^{(j)} T^{j}$ with $E^{(j)}=F_{(j+1)}$ to obtain $G(T)$, $H(T)$ in $E A_{\mathrm{dn}}^{0}(k[T])$ such that $E^{\prime}(T)=G(T) \circ E(T)^{[(d-1) n]} \circ H(T)$ has the form $E^{\prime}(T)=X_{\mathrm{dn}}+N T$ with $N$ as above. Setting $T=1$ we obtain $F^{\prime}=E^{\prime}(1)$ $=G(1) \circ F^{[(d-1) n]} \circ H(1)$ with $G(1), H(1) \in E A_{\mathrm{dn}}^{0}(k)$.

Suppose that $J(F)$ is invertible. We have $J(E)=\Sigma_{j} J\left(F_{(j)}\right) T^{j-1}$. Since $J\left(F_{(j)}\right)$ is homogeneous of degree $j-1$ in $X$ we have $J(E)(T, X)=J(F)(T X)$; hence $J(E)$ is invertible. Therefore $J\left(E^{\prime}\right)=I+J(N) T$ is also invertible. Applying Lemma (4.1) as in $\S 4$ we conclude that $J(N)$ is nilpotent. 
6. Nilpotent rank 1 Jacobians. Theorem (6.2) below affirms the Jacobian Conjecture for maps $F=X-H$ when $J(H)$ is nilpotent of rank 1 . This result was obtained in collaboration with M. P. Murthy, to whom we are grateful.

(6.1) Proposition. Let $k$ be a field of characteristic zero. Let $T \in k^{[n]}$ and let $r$ denote the dimension of the $k$-vector space generated by $D_{1} T, \ldots, D_{n} T$. There is a special linear change of variables $X_{i} \mapsto L_{i}$ such that, if $S=T(L)$, then $S$ depends only on $X_{1}, \ldots, X_{r}$, and $D_{1} S, \ldots, D_{r} S$ are linearly independent over $k[S]$.

$$
\begin{aligned}
& \text { If } L_{j}=\Sigma_{i} L_{i j} X_{i} \text {, each } L_{i j} \in k \text {, then } \\
& \qquad D_{i} S=\sum_{j}\left(D_{j} T\right)(L) \cdot\left(D_{i} L_{j}\right)=\sum_{j}\left(L_{i j}\left(D_{j} T\right)\right)(L) .
\end{aligned}
$$

Thus we can choose $\left(L_{i j}\right) \in S L_{n}(k)$ so that $D_{1} S, \ldots, D_{r} S$ are linearly independent over $k$, and $D_{r+1} S=\cdots=D_{n} S=0$, so that (since $\operatorname{char}(k)=0$ ) $S$ depends only on $X_{1}, \ldots, X_{r}$. Suppose, finally, that there is a relation $\sum_{i=1}^{r} P_{i}(S)\left(D_{i} S\right)=0$ with each $P_{i}(S) \in k[S]$ and with some $P_{i} \neq 0$. We may assume that $P_{1}, \ldots, P_{r}$ have g.c.d. 1 in $k[S]$, so that, for $S=0$, some $P_{i}(0) \neq 0$. Then we have a nontrivial congruence $\Sigma_{i=1}^{r} P_{i}(0)\left(D_{i} S\right) \equiv 0 \bmod S \cdot k^{[n]}$. Since the left side has $X$-degree $<\operatorname{deg}(S)$, we must have $\sum_{i=1}^{r} P_{i}(0)\left(D_{i} S\right)=0$, contradicting the $k$-linear independence of $D_{1} S, \ldots, D_{r} S$. This proves the Proposition.

(6.2) THEOREM. Let $k$ be a field of characteristic zero. Suppose that $F \in$ $\operatorname{End}\left(\mathbf{A}_{k}^{n}\right)$ is of the form $F=X-H$ where $H(0)=0$ and $J(H)$ is nilpotent of rank 1 .

(a) The integral closure of $k\left[H_{1}, \ldots, H_{n}\right]$ in $k^{[n]}$ is a polynomial ring $k[T]$ for some $T \in k^{[n]}$.

(b) There is a linear automorphism $L$ of $\mathbf{A}_{k}^{n}$ such that if $S=T(L)$ then, for some $r \geqslant 1, S$ depends only on $X_{1}, \ldots, X_{r}$ and $D_{1} S, \ldots, D_{r} S$ are linearly independent over $k[S]$.

(c) Put $F^{\prime}=L^{-1} \circ F \circ L$. Then $F^{\prime}=X-K$ where each $K_{i}$ depends only on $X_{1}, \ldots, X_{r}\left(\right.$ in fact $\left.K_{i} \in k[S]\right)$ and $K_{i}=0$ for $i \leqslant r$.

(d) $F^{\prime}$ is a product of elementary automorphisms. In particular $F$ is invertible.

(6.3) Corollary. Let $k$ be a field of characteristic zero and let $F \in \operatorname{End}\left(\mathbf{A}_{k}^{n}\right)$ be of the form $F=X-H$ with $H(0)=0$ and $J(H)$ nilpotent. If $n=2$ or if $n=3$ and $J(H)^{2}=0$ then $F$ is a product of elementary automorphisms and, in particular, is invertible.

Indeed under the conditions of the Corollary $J(H)$ must have rank 1.

Proof of Theorem (6.2). From the exact sequence

$$
k(X) \otimes_{k(H)} \Omega_{k(H) / k} \rightarrow \Omega_{k(X) / k} \rightarrow \Omega_{k(X) / k(H)} \rightarrow 0
$$

and the condition, $\operatorname{rank} J(H)=1$, we conclude that $\operatorname{rank}_{k(X)} \Omega_{k(X) / k(H)}=$ $n-1$. Since char $k=0$ it follows that the transcendence degree of $k(X) / k(H)$ is $n-1$ (cf. [Mat], (27.B)), and so that of $k(H) / k$ is 1 . It follows therefore from [A $+\mathbf{H}+\mathbf{E}],(2.6)$, that the integral closure of $k[H]$ in $k[X]$ is a polynomial ring $k[T]$, whence (a). Now (b) follows from Proposition (6.1). 
Writing $H=Q(T)$, where $Q=\left(Q_{1}, \ldots, Q_{n}\right)$, each $Q_{i} \in k[T]$, we have $F^{\prime}=X$ - $K$ where $K=L^{-1} \cdot H \cdot L=L^{-1}(Q)(S)$, and where $S=T(L)$. Let $L^{-1}(Q)$ $=R=\left(R_{1}, \ldots, R_{n}\right)$, so that $K_{i}=R_{i}(S) \in k\left[X_{1}, \ldots, X_{r}\right]$. The Jacobian of $K$ is the rank 1 matrix

$$
J(K)=\left(\begin{array}{c}
R_{1}^{\prime}(S) \\
\vdots \\
R_{n}^{\prime}(S)
\end{array}\right) \cdot\left(D_{1} S, \ldots, D_{r} S, 0, \ldots, 0\right)
$$

which, being nilpotent, has trace

$$
\operatorname{Tr}(J(K))=\sum_{i=1}^{r} R_{i}^{\prime}(S)\left(D_{i} S\right)=0 .
$$

Since $D_{1} S, \ldots, D_{r} S$ are linearly independent over $k[S]$ we must have $R_{1}^{\prime}(S)=$ $\cdots=R_{r}^{\prime}(S)=0$. Therefore $(\operatorname{char}(k)=0) R_{1}(S), \ldots, R_{r}(S)$ are constants. But since $K(0)=0$ we have $R_{i}(0)=0$ for all $i \leqslant r$, and so $R_{1}=\cdots=R_{r}=0$. This proves (c). It is clear that $F^{\prime}$ is the product of the (commuting) elementary automorphisms $E^{(j)}(j=r+1, \ldots, n)$ defined by $E_{i}^{(j)}=X_{i}$ for $i \neq j$ and $E_{j}^{(j)}=X_{j}-K_{j}$. This proves (d), and completes the proof of the theorem.

\section{The Formal InVerse}

The main result of this chapter is the "tree expansion" (Theorem (4.1)) of the polynomial $G_{i}^{(d)}$. We start with power series $F_{i}=X_{i}-H_{i}$, where $H_{i}$ involves only terms of degree $\geqslant 2, i=1, \ldots, n$. Then $G_{i}$ is defined by $G_{i}(F)=$ $X_{i}$, and we have a decomposition $G_{i}=\Sigma_{d>0} G_{i}^{(d)}$. The chapter begins with a formula of Abhyankar [Ab2] for $G_{i}$ (Corollary (2.2)).

1. Notation. Let $k$ be a commutative Q-algebra. For each integer $n \geqslant 0$ we put

$$
\mathbf{n}=\{1, \ldots, n\}, \quad X_{\mathbf{n}}=\left(X_{1}, \ldots, X_{n}\right),
$$

a sequence of indeterminates,

$$
k^{[n]}=k\left[X_{1}, \ldots, X_{n}\right] \quad \text { and } k^{[[n]]}=k\left[\left[X_{1}, \ldots, X_{n}\right]\right],
$$

the power series algebra. Put

$$
M A_{n}^{0}((k))=\left\{F=\left(F_{1}, \ldots, F_{n}\right) \mid F_{i} \in k^{[[n]]}, \quad F_{i}(0)=0, i=1, \ldots, n\right\} .
$$

If $U \in k^{[[n]]}$ and $F \in M A_{n}^{0}((k))$ we put

$$
\varphi_{F}(U)=U(F)=U\left(F_{1}, \ldots, F_{n}\right) .
$$

Then $\varphi_{F}$ is the $k$-algebra endomorphism of $k^{[[n]]}$ such that $\varphi_{F}\left(X_{i}\right)=F_{i}$ $(i=1, \ldots, n)$. If $G \in M A_{n}^{0}((k))$ then $\varphi_{G} \circ \varphi_{F}=\varphi_{F(G)}$ where $F(G)=$ $\left(F_{1}(G), \ldots, F_{n}(G)\right)$. Thus $M A_{n}^{0}((k))$, with the composition $F \circ G=F(G)$, is a monoid anti-isomorphic to a monoid of $k$-algebra endomorphisms of $k^{[[n]]}$. The neutral element is $X\left(=X_{\mathfrak{n}}\right)$. Its group of invertible elements will be denoted $G A_{n}^{0}((k))$. 
The Jacobian matrix of $F \in M A_{n}^{0}((k))$ is

$$
J(F)=\left(\begin{array}{ccc}
D_{1} F_{1} & \cdots & D_{n} F_{1} \\
\vdots & & \vdots \\
D_{1} F_{n} & \cdots & D_{n} F_{n}
\end{array}\right) \quad\left(D_{i}=\frac{\partial}{\partial X_{i}}\right)
$$

and its Jacobian determinant is

$$
j(F)=\operatorname{det} J(F) .
$$

We have $J(G(F))=J(G)(F) \cdot J(F)$, and so also $j(G(F))=j(G)(F) \cdot j(F)$. We have a monoid homomorphism

$$
J_{0}: M A_{n}^{0}((k)) \rightarrow M_{n}(k), \quad J_{0}(F)=J(F)(0),
$$

and $G A_{n}^{0}((k))$ consists of those $F$ for which $J_{0}(F)$ is invertible. We have a split exact sequence

$$
1 \rightarrow G A_{n}^{1}((k)) \rightarrow G A_{n}^{0}((k)) \underset{L}{\stackrel{J_{0}}{\rightleftarrows}} G L_{n}(k) \rightarrow 1
$$

where, if $\alpha=\left(a_{i j}\right) \in G L_{n}(k)$, then $L(\alpha)=\left(L_{1}, \ldots, L_{n}\right)$ with $L_{i}=\Sigma_{j} a_{i j} X_{j}$ $(i=1, \ldots, n)$. The kernel $G A_{n}^{1}((k))$ of $J_{0}$ consists of those $F$ of the form $X+H$ where $H$ involves only monomials of degree $\geqslant 2$ in $X$.

The aim of this chapter is to study the inverse $G$ of an element $F \in G A_{n}^{1}((k))$.

2. Abhyankar's Inversion Formula, $G_{i}=\Sigma_{p} D^{[p]}\left(X_{i} \cdot j(F) \cdot(X-F)^{p}\right)$.

Let $\mathbf{N}=\{0,1,2,3, \ldots\}$. If $p=\left(p_{1}, \ldots, p_{n}\right) \in \mathbf{N}^{n}$ put

$$
p !=p_{1} ! \cdots p_{n} !
$$

(where $0 !=1$ ). For any commutative Q-algebra $A$ and $a=\left(a_{1}, \ldots, a_{n}\right) \in A^{n}$ we put

$$
a^{p}=a p_{1}^{p_{1}} \cdots a_{n}^{p_{n}} \text { and } a^{[p]}=a^{p} / p !
$$

(By convention $a^{0}=1$ even if all $a_{i}=0$.) If $a, b \in A^{n}$ we can rewrite the Binomial Theorem,

$$
(a+b)^{p}=\sum_{q+r=p} \frac{p !}{q ! r !} a^{q} b^{r},
$$

in the form

$$
(a+b)^{[p]}=\sum_{q+r=p} a^{[q]} b^{[r]}
$$

Let $F \in G A_{n}^{1}((k))$, so that $F_{i}=X_{i}+$ terms of degree $\geqslant 2$ for $i=1, \ldots, n$. For each $U \in k^{[[n]]}$ put

$$
\begin{aligned}
\langle U, F\rangle & =\sum_{p \in \mathbf{N}^{n}} D^{[p]}\left(U(F) \cdot J(F) \cdot(X-F)^{p}\right) \\
& =\sum_{p \in \mathbf{N}^{n}} D^{p}\left(U(F) \cdot j(F) \cdot(X-F)^{[p]}\right) .
\end{aligned}
$$


Recall here that,

$$
D^{[p]}=\frac{D p_{1} \cdots D_{n}^{p_{n}}}{P_{1} ! \cdots p_{n} !} \quad \text { and } \quad(X-F)^{p}=\left(X_{1}-F_{1}\right)^{p_{1}} \cdots\left(X_{n}-F_{n}\right)^{p_{n}} \text {. }
$$

(2.1) TheOREm (ABHyANKAR [Ab2]). With the notation above, we have $\langle U, F\rangle=U$.

Putting $H=X-F$ we have

$$
\langle U, F\rangle=\sum_{p} D^{[p]}\left(U(F) \cdot j(F) \cdot H^{p}\right)
$$

(2.2) Corollary. Let $G=\left(G_{1}, \ldots, G_{n}\right)$ be the inverse of $F: G_{i}(F)=X_{i}$ for $i=1, \ldots, n$. Then

$$
G_{i}=\sum_{p \in \mathbf{N}^{n}} D^{[p]}\left(X_{i} \cdot j(F) \cdot H^{p}\right)
$$

(2.3) COROllary. We have

$$
1=\sum_{p \in \mathbf{N}^{n}} D^{[p]}\left(j(F) \cdot H^{p}\right)
$$

so that

$$
1-j(F)=\sum_{p \neq 0} D^{[p]}\left(j(F) \cdot H^{p}\right) .
$$

Further we have

$$
X_{i}=\sum_{p \in \mathbf{N}^{n}} D^{[p]}\left(F_{i} \cdot j(F) \cdot H^{p}\right) .
$$

(2.4) Corollary. For $U(X) \in k^{[[n]]} p u t$

$$
V(X)=\sum_{p \in \mathbf{N}^{n}} D^{[p]}\left(U(X) \cdot j(F) \cdot H^{p}\right) .
$$

Then

$$
U(X)=V(F) .
$$

In fact, if we define $V$ by the second formula then, by Theorem (2.1), it is given by the first formula.

Corollary (2.4) expresses each element of $k^{[[n]]}$ as a power series in $F$. By taking the Taylor expansion of $V(X)$ and substituting $F$ for $X$ we obtain the following formula.

(2.5) COROllaRy (GURJAR). For $U \in k^{[[n]]}$ we have

$$
U(X)=\sum_{q \in \mathbf{N}^{n}} \sum_{p \in \mathbf{N}^{n}}\left[D^{p+q}\left(U(X) \cdot j(F) \cdot H^{[p]}\right)\right]_{X=0} \cdot F^{[q]} .
$$

In fact it was Gurjar's discovery of (2.5), based on a formula of Goursat in the case of two variables, that led Abhyankar to the discovery of Theorem (2.1). 
(2.6) REMARK. Writing $\langle U, F\rangle=\Sigma_{p} D^{[p]}\left(U(F) \cdot j(F) \cdot H^{p}\right)$ makes sense for an arbitrary commutative ring $k$, not necessarily a $\mathbf{Q}$-algebra, by defining

$$
D^{[p]} X^{q}=\left(\begin{array}{l}
q_{1} \\
p_{1}
\end{array}\right) \cdots\left(\begin{array}{l}
q_{n} \\
p_{n}
\end{array}\right) X^{q-p}
$$

which is zero if $q_{i}<p_{i}$ for any $i$. Moreover it remains true that $\langle U, F\rangle=U$. In fact let $A$ be a polynomial ring over $\mathbf{Z}$ in indeterminates $C_{i, p}$ and $U_{p}\left(p \in \mathbf{N}^{n}\right.$; $i=1, \ldots, n)$. Put $F_{i}=X_{i}+\Sigma_{|p|>2} C_{i, p} X^{p} \quad(i=1, \ldots, n)$ and $U=\Sigma_{p} U_{p} X^{p}$. Then $F=\left(F_{1}, \ldots, F_{n}\right) \in G A_{n}^{1}((A))$. By embedding $A$ in $\mathbf{Q} \otimes_{\mathbf{Z}} \mathbf{A}$ and applying Theorem (2.1) we conclude that $\langle U, F\rangle=U$. The formula in general follows now by "specializing" this generic example.

Theorem (2.1) will be proved as in Abhyankar [Ab2], in several steps.

Step 1. Suppose that $F=H \circ G$, i.e. $F=H(G)$. If the Theorem is valid for $G$ and $H$ then it is valid for $F$.

We have

$$
\begin{aligned}
\langle U, F\rangle=\sum_{p} D^{p}\left(U(H(G)) \cdot j(H(G)) \cdot(X-H(G))^{[p]}\right) \\
=\sum_{p} D^{p}\left(U(H(G)) \cdot j(G) \cdot j(H)(G) \cdot(X-G+G-H(G))^{[p]}\right) \\
=\sum_{p} D^{p}\left(U(H(G)) \cdot j(H)(G) \cdot j(G) \cdot \sum_{q+r=p}(G-H(G))^{[q]} \cdot(X-G)^{[r]}\right) \\
=\sum_{q} D^{q}\left(\sum_{r} D^{r}\left(U(H(G)) \cdot j(H)(G) \cdot(G-H(G))^{[q]} \cdot j(G) \cdot(X-G)^{[r]}\right)\right) \\
=\sum_{q} D^{q}\left\langle V_{q}, G\right\rangle \quad\left[V_{q}=U(H) j(H)(X-H)^{[q]}\right] \\
=\sum_{q} D^{q} V_{q}=\langle U, H\rangle=U,
\end{aligned}
$$

where the last equalities invoke the assumptions on $G$ and $H$, respectively.

Step 2. $F=H^{(1)} \circ \ldots \circ H^{(n)}$ where $H^{(i)}=\left(X_{1}, \ldots, X_{i-1}, G_{i}, X_{i+1}, \ldots, X_{n}\right)$ alters only the variable $X_{i}$.

Indeed, define $G_{i}$ by the condition

$$
G_{i}\left(X_{1}, \ldots, X_{i}, F_{i+1}, \ldots, F_{n}\right)=F_{i} .
$$

Since $F \in G A_{n}^{1}((k))$ there is a unique such $G_{i}$, and then $H^{(i)}$ as above is again in $G A_{n}^{1}((k))$. Moreover $G_{n}=F_{n}$ and it follows by induction on $n-i$ that

$$
H^{(i)} \circ \ldots \circ H^{(n)}=\left(X_{1}, \ldots, X_{i-1}, F_{i}, \ldots, F_{n}\right),
$$

whence the claim above.

Step 3. In view of Steps 1 and 2 it suffices to prove the Theorem for an $F$ that alters only one of the variables, say $X_{1}$.

$$
F=\left(F_{1}, X_{2}, \ldots, X_{n}\right) \text {. }
$$


Then $j(F)=D_{1} F_{1}$, clearly. Moreover, for $p \in \mathbf{N}^{n},(X-F)^{[p]}=0$ unless $p$ has the form $\left(p_{1}, 0, \ldots, 0\right)$, when $(X-F)^{[p]}=\left(X_{1}-F_{1}\right)^{\left[p_{1}\right]}$. Thus

$$
\langle U, F\rangle=\sum_{p_{1}=0}^{\infty} D_{1}^{p_{1}}\left(U(F) D_{1} F_{1}\left(X_{1}-F_{1}\right)^{\left[p_{1}\right]}\right) .
$$

Write

$$
U=\sum_{m=0}^{\infty} U_{m}\left(X_{2}, \ldots, X_{n}\right) X_{1}^{m}
$$

with each $U_{m} \in k\left[\left[X_{2}, \ldots, X_{n}\right]\right]$. Then

$$
\begin{aligned}
\langle U, F\rangle & =\sum_{m=0}^{\infty} U_{m}\left(X_{2}, \ldots, X_{n}\right) \sum_{p_{1}=0}^{\infty} D_{1}^{p_{1}}\left(F_{1}^{m} D_{1} F_{1}\left(X_{1}-F_{1}\right)^{\left[p_{1}\right]}\right) \\
& =\sum_{m=0}^{\infty} U_{m}\left(X_{2}, \ldots, X_{n}\right)\left\langle X_{1}^{m}, F_{1}\right\rangle .
\end{aligned}
$$

It remains to show that $\left\langle X_{1}^{m}, F_{1}\right\rangle=X_{1}^{m}$. Thus we have reduced the proof to the case of:

Step 4. Assume that $n=1$. Write $X$ for $X_{1}$ and $D$ for $d / d X$. Let $F=X-H$ with $H \in X^{2} k[[X]]$. We claim that, for each $m \geqslant 0$,

$$
X^{m}=\sum_{p=0}^{\infty} D^{[p]}\left((X-H)^{m}(1-D H) H^{p}\right)
$$

where $D^{[p]}=D^{p} / p$ !. Recall Leibniz' Rule,

$$
\begin{gathered}
D^{[p]}(R S)=\sum_{u+v=p} D^{[u]} R \cdot D^{[v]} S . \\
\sum_{p=0}^{\infty} D^{[p]}\left((X-H)^{m}(1-D H) H^{p}\right) \\
=\sum_{p=0}^{\infty} D^{[p]} \sum_{i=0}^{m}(-1)^{i}\left(\begin{array}{c}
m \\
i
\end{array}\right) X^{m-i}\left(H^{p+i}-H^{p+i} D H\right) \\
=\sum_{p=0}^{\infty} \sum_{i=0}^{m} \sum_{j=0}^{\min (p, m-i)}(-1)^{i}\left(\begin{array}{c}
m \\
i
\end{array}\right) D^{[j]} X^{m-i} \cdot D^{[p-j]}\left(H^{p+i}-H^{p+i} D H\right) \\
=\sum_{p=0}^{\infty} \sum_{i=0}^{m} \sum_{j=0}^{\min (p, m-i)}(-1)^{i} \frac{m !(m-i) !}{i !(m-i) ! j !(p-j) !(m-i-j) !} \\
\cdot X^{m-i-j} \cdot D^{p-j}\left(H^{p+i}-H^{p+i} D H\right) .
\end{gathered}
$$


Set $t=i+j$ and $q=p-j$. Then this last expression equals

$$
\begin{aligned}
& \sum_{t=0}^{m} \sum_{i=0}^{t} \sum_{q=0}^{\infty}(-1)^{i} \frac{m !}{i !(t-i) ! q !(m-t) !} X^{m-t} D^{q}\left(H^{q+t}-H^{q+t} D H\right) \\
& =\sum_{t=0}^{m} \sum_{i=0}^{t} \sum_{q=0}^{\infty}(-1)^{i}\left(\begin{array}{c}
t \\
i
\end{array}\right)\left(\begin{array}{c}
m \\
t
\end{array}\right) X^{m-t} D^{[q]}\left(H^{q+t}-H^{q+t} D H\right) \\
& =\sum_{t=0}^{m}\left(\begin{array}{c}
m \\
t
\end{array}\right) X^{m-t}\left(\sum_{i=0}^{t}(-1)^{i}\left(\begin{array}{c}
t \\
i
\end{array}\right)\right) \cdot\left(\sum_{q=0}^{\infty} D^{[q]}\left(H^{q+t}-H^{q+t} D H\right)\right) .
\end{aligned}
$$

Now $\sum_{i=0}^{t}(-1)^{i}\left(\begin{array}{l}t \\ i\end{array}\right)=(1-1)^{t}$ vanishes for $t>0$, and is 1 for $t=0$. Moreover

$$
\begin{aligned}
\left(\sum_{q=0}^{\infty} D^{[q]}\left(H^{q+t}-H^{q+t} D H\right)\right)_{t=0} & =\sum_{q=0}^{\infty}\left(D^{[q]} H^{q}-D^{[q]} D\left(\frac{H^{q+1}}{q+1}\right)\right) \\
& =\sum_{q=0}^{\infty}\left(D^{[q]} H^{q}-D^{[q+1]} H^{q+1}\right)=1 .
\end{aligned}
$$

This proves the claim of Step 4, and thus proves the Theorem.

3. The terms $G_{i}^{(d)}$. We fix $F=X-H \in G A_{n}^{1}((k))$ satisfying:

(1) $H_{1}, \ldots, H_{n}$ are homogeneous polynomials of the same degree $\delta(\geqslant 2)$;

$$
\begin{gathered}
J(H) \text { is nilpotent; and } \\
j(F)(=\operatorname{det} J(F))=1 .
\end{gathered}
$$

To prove the Jacobian Conjecture we can assume that these conditions hold with $\delta \leqslant 3$ (Corollary (2.2) of Chapter II).

We have $J(F)=I-J(H)$ with $J(H)$ homogeneous in $X$ of degree $\delta-1$. Applying Lemma (4.1) of Chapter II to $A=M_{n}(k)[X]$ and $a=-J(H) \in A_{\delta-1}$ we see that (2) is actually a consequence of (3). The converse holds if $k$ is reduced, e.g. a field.

For all $U \in k^{[[n]]}$ we have (Theorem (2.1))

$$
U=\sum_{p \in \mathbf{N}^{n}} D^{[p]}\left(U(F) \cdot H^{p}\right)
$$

In particular the inverse series $G_{i}$ defined by $G_{i}(F)=X_{i}$ is given (Corollary (2.2)) by

$$
G_{i}=\sum_{d=0}^{\infty} G_{i}^{(d)}
$$

where

$$
G_{i}^{(d)}=\sum_{|p|=d} D^{[p]}\left(X_{i} \cdot H^{p}\right) .
$$

(Recall that $|p|=p_{1}+\cdots+p_{n}$ for $p \in \mathbf{N}^{n}$.) Each $G_{i}^{(d)}$ is a homogeneous polynomial of degree $(1+d \delta)-d=d(\delta-1)+1$. It follows that

$$
G_{i} \text { is a polynomial iff } G_{i}^{(d)}=0 \text { for all } d \gg 0 \text {. }
$$


In fact, assuming that $k$ is a field, it follows from Corollary (1.4) of Chapter I that we must then have $G_{i}^{(d)}=0$ as soon as $d(\delta-1)+1>\delta^{n-1}$. Thus, if $k$ is a field,

(7) $\quad G_{i}$ is a polynomial iff $G_{i}^{(d)}=0$ for $d>\left(\delta^{n-1}-1\right) /(\delta-1)$.

These remarks motivate the following detailed study of the expressions $G_{i}^{(d)}$. (3.1) Notation. For every $L \in k^{[[n]]}$ and $d \geqslant 0$ we put

$$
L_{[d]}=d ! \sum_{|p|=d} D^{[p]}\left(L \cdot H^{p}\right)
$$

For example:

$$
\begin{gathered}
L_{[0]}=L, \\
L_{[1]}=\sum_{p=1}^{n} D_{p}\left(L H_{p}\right)=\sum_{p=1}^{n}\left(D_{p} L\right) H_{p}+L \cdot \sum_{p=1}^{n} D_{p} H_{p} \\
=\sum_{p=1}^{n}\left(D_{p} L\right) H_{p}+L \cdot \operatorname{Tr}(J(H))=\sum_{p=1}^{n}\left(D_{p} L\right) \cdot H_{p},
\end{gathered}
$$

in view of conditions (1) and (3).

(2)

$$
1_{[d]}=0 \text { for } d>0 \text {, }
$$

since $1=\Sigma_{p} D^{[p]}\left(H^{p}\right)($ Corollary (2.3)).

$$
G_{i}^{(d)}=\frac{1}{d !} X_{i[d]} \quad(\text { formula }(5)) .
$$

In particular

$$
G_{i}^{(0)}=X_{i} \quad(\text { by }(0))
$$

and

$$
G_{i}^{(1)}=H_{i} \quad(\text { by }(1)) .
$$

(3.2) LEMMA. For $L \in k^{[[n]]}$ and $d \geqslant 0$ we have

$$
L_{[d]}=\sum_{r: \mathbf{d} \rightarrow \mathbf{n}} D_{r}\left(L \cdot H_{r}\right) .
$$

Here, and in what follows, if $r: \mathbf{d} \rightarrow \mathbf{n}$ and $r_{i}=r(i)$, we put $D_{r}=D_{r_{1}} \cdots D_{r_{d}}$ and $H_{r}=H_{r_{1}} \cdots H_{r_{d}}$.

PROOF. To each $r: \mathbf{d} \rightarrow \mathbf{n}$ we associate $p(r)=\left(p_{1}(r), \ldots, p_{n}(r)\right)$ where $p_{i}(r)$ $=\operatorname{Card}\left(r^{-1}(i)\right)$. Note then that $|p(r)|=d, D_{r}=D^{p(r)}$, and $H_{r}=H^{p(r)}$. If $p \in \mathbf{N}^{n}$ and $|p|=d$ put $N_{p}=\operatorname{Card}\{r: \mathbf{d} \rightarrow \mathbf{n} \mid p(r)=p\}$. Then

$$
\begin{aligned}
\sum_{r: \mathbf{d} \rightarrow \mathbf{n}} D_{r}\left(L \cdot H_{r}\right) & =\sum_{|p|=d} N_{p} \cdot D^{p}\left(L \cdot H^{p}\right) \\
& =\sum_{|p|=d} N_{p} \cdot p ! \cdot D^{[p]}\left(L \cdot H^{p}\right) .
\end{aligned}
$$

It suffices therefore to show that $N_{p} \cdot p !=d !$. 
The symmetric group $S_{d}$ acts on $\{r: \mathbf{d} \rightarrow \mathbf{n}\}$ with orbits the fibers of the map $r \mapsto p(r)$. Let $T_{p} \subset S_{d}$ denote the stabilizer of some $r$ such that $p(r)=p$. It is easy to see that $T_{p}$ is isomorphic to $S_{p_{1}} \times \cdots \times S_{p_{n}}$. Thus $N_{p}=\left[S_{d}: T_{p}\right]=$ $d ! / p !$, as required.

(3.3) Lemma. For $L \in k^{[[n]]}$ and $d>0$,

$$
L_{[d]}=\sum_{e=1}^{d}\left(\begin{array}{l}
d \\
e
\end{array}\right) \sum_{f: \mathbf{e} \rightarrow \mathbf{n}}\left(D_{f} L\right) \cdot\left(H_{f}\right)_{[d-e]} .
$$

If $S \subset \mathbf{d}$ put $S^{\prime}=\mathbf{d}-S$ and, for $r: \mathbf{d} \rightarrow \mathbf{n}, D_{r_{S}}=\prod_{i \in S} D_{r_{i}}$. Then, from (3.2),

$$
\begin{aligned}
L_{[d]} & =\sum_{r: \mathbf{d} \rightarrow \mathbf{n}} D_{r}\left(L \cdot H_{r}\right) \\
& =\sum_{r: \mathbf{d} \rightarrow \mathbf{n}} \sum_{S \subset \mathbf{d}}\left(D_{r_{s}} L\right)\left(D_{r_{s^{\prime}}} H_{r}\right) \\
& =\sum_{S \subset \mathbf{d}}\left(\sum_{f: S \rightarrow \mathbf{n}} \sum_{g: S^{\prime} \rightarrow \mathbf{n}}\left(D_{f} L\right) D_{g}\left(H_{f} H_{g}\right)\right) \\
& =\sum_{e=0}^{d}\left(\begin{array}{l}
d \\
e
\end{array}\right) \sum_{f: \mathbf{e} \rightarrow \mathbf{n}}\left(D_{f} L\right)\left(\sum_{g: \mathbf{d}-\mathbf{e} \rightarrow n} D_{g}\left(H_{f} H_{g}\right)\right) \\
& =\sum_{e=0}^{d}\left(\begin{array}{l}
d \\
e
\end{array}\right) \sum_{f: \mathbf{e} \rightarrow \mathbf{n}}\left(D_{f} L\right) \cdot\left(H_{f}\right)_{[d-e]} .
\end{aligned}
$$

For $e=0$ we have $H_{f}=1$, and $1_{[d]}=0$ for $d>0$ ((3.1), Example (2)); whence the lemma.

(3.4) LeMma. For $L \in k^{[[n]]}$ and $d>0$ we have

$$
L_{[d]}=\sum_{h \geqslant 1} \sum_{\substack{e \in \mathbb{d}^{h} \\
|e|=d}}\left(\begin{array}{l}
d \\
e
\end{array}\right) \sum_{\substack{f=\left(f_{1}, \ldots, f_{n}\right) \\
f_{j}: \mathbf{e}_{j} \rightarrow \mathbf{n}}} L_{e, f}
$$

where $|e|=e_{1}+\cdots+e_{h}$,

$$
\left(\begin{array}{l}
d \\
e
\end{array}\right)=\frac{d !}{e !}=\frac{d !}{e_{1} ! \cdots e_{h} !},
$$

and where

$$
L_{e, f}=\left(D_{f_{1}} L\right)\left(D_{f_{2}} H_{f_{1}}\right) \cdots\left(D_{f_{h}} H_{f_{h-1}}\right) H_{f_{h}} .
$$

We argue by induction on $d$. For $d=1$ it is (3.1), Example (1), $L_{[1]}=$ $\sum_{j=1}^{n}\left(D_{j} L\right) H_{f}$. Suppose,

Example (1), $L_{[1]}=\sum_{f=1}^{n}\left(D_{f} L\right) \cdot H_{f}$. Suppose that $d \geqslant 2$. By Lemma (3.3),

$$
L_{[d]}=\sum_{e_{1}=1}^{d}\left(\begin{array}{c}
d \\
e_{1}
\end{array}\right) \sum_{f_{1}: \mathbf{e}_{1} \rightarrow \mathbf{n}}\left(D_{f_{1}} L\right) \cdot\left(H_{f_{1}}\right)_{\left[d-e_{1}\right]}
$$


By induction

$$
\left(H_{f_{1}}\right)_{\left[d-e_{1}\right]}=\sum_{h \geq 2} \sum_{\substack{e=\left(e_{2}, \ldots, e_{h}\right) \\
e_{2}+\cdots+e_{h}=d-e_{1}}}\left(\begin{array}{c}
d-e_{1} \\
e_{2}, \ldots, e_{h}
\end{array}\right) \sum_{\substack{f=\left(f_{2}, \ldots, f_{h}\right) \\
f_{j}: \mathbf{e}_{j} \rightarrow \mathbf{n}}}\left(H_{f_{1}}\right)_{e, f} .
$$

Now

$$
\left(\begin{array}{c}
d \\
e_{1}
\end{array}\right)\left(\begin{array}{c}
d-e_{1} \\
e_{2}, \ldots, e_{h}
\end{array}\right)=\frac{d ! \cdot\left(d-e_{1}\right) !}{e_{1} !\left(d-e_{1}\right) ! \cdot e_{2} ! \cdots e_{h} !}=\frac{d !}{e_{1} ! \cdots e_{h} !}
$$

and

$$
\left(D_{f_{1}} L\right) \cdot\left(H_{f_{1}}\right)_{e, f}=\left(D_{f_{1}} L\right)\left(D_{f_{2}} H_{f_{1}}\right) \cdots\left(D_{f_{h}} H_{f_{h-1}}\right) H_{f_{h}}
$$

whence the lemma.

(3.5) Proposition. We have $G_{i}^{(0)}=X_{i}, G_{i}^{(1)}=H_{i}$, and, for $d \geqslant 2$,

$$
d ! G_{i}^{(d)}=\sum_{h=2}^{d} \sum_{\substack{e=\left(e_{2}, \ldots, e_{h}\right) \\
1+e_{2}+\cdots+e_{h}=d}}\left(\begin{array}{c}
d \\
1, e_{2}, \ldots, e_{h}
\end{array}\right) \sum_{\substack{f=\left(f_{2}, \ldots, f_{h}\right) \\
f_{j}: \mathbf{e}_{j} \rightarrow \mathbf{n}}}\left(H_{i}\right)_{e, f}
$$

where

$$
\left(\begin{array}{c}
d \\
1, e_{2}, \ldots, e_{h}
\end{array}\right)=\frac{d !}{e_{2} ! \cdots e_{h} !}
$$

and

$$
\left(H_{i}\right)_{e, f}=\left(D_{f_{2}} H_{i}\right)\left(D_{f_{3}} H_{f_{2}}\right) \cdots\left(D_{f_{h}} H_{f_{h-1}}\right) H_{f_{h}} .
$$

The first formulas follow from (3.1) Example (3). The last formula follows from (3.4) with $L=X_{i}$. Then $D_{f_{1}} X_{i}=0$ unless $e_{1}=1$ and $f_{1}=i$.

4. The tree expansion $G_{i}^{(d)}=\Sigma_{T}(1 / \alpha(T)) \Sigma_{f} P_{T, f}$. To pursue the expansion of Proposition (3.5) we must develop expressions of the form $D_{f} H_{g}$ where $f$ : $\mathbf{e} \rightarrow \mathbf{n}$ and $g: \mathbf{e}^{\prime} \rightarrow \mathbf{n}$. We have

$$
D_{f} H_{g}=\sum_{u: \mathbf{e} \rightarrow \mathbf{e}^{\prime}}\left(D_{f, u^{-1}(1)} H_{g(1)}\right) \cdots\left(D_{f, u^{-1}\left(e^{\prime}\right)} H_{g\left(e^{\prime}\right)}\right)
$$

where, for $S \subset \mathbf{e}, D_{f, S}=\prod_{i \in S} D_{f(i)}$. Applying (1) to the expressions $D_{f_{j}} H_{f_{j-1}}$ in (3.5) we obtain

$$
\left(H_{i}\right)_{e, f}=\sum_{u}\left(H_{i}\right)_{e, f, u}
$$

where $u$ varies over $(h-1)$-tuples $u=\left(u_{2}, \ldots, u_{h}\right)$ with $u_{j}: \mathbf{e}_{j} \rightarrow \mathbf{e}_{j-1}(j=$ $2, \ldots, h ; e_{1}=1$ by convention), and where

$$
\text { (3) } \begin{aligned}
\left(H_{i}\right)_{e, f, u}= & \left(D_{f_{2}} H_{i}\right) \cdot\left[\left(D_{f_{3}, u_{3}^{-1}(1)} H_{f_{2}(1)}\right) \cdots\left(D_{f_{3}, u_{3}^{-1}\left(e_{2}\right)} H_{f_{2}\left(e_{2}\right)}\right)\right] \\
& \cdots\left[\left(D_{f_{h}, u_{h}^{-1}(1)} H_{f_{h-1}(1)}\right) \cdots\left(D_{f_{h}, u_{h}^{-1}\left(e_{h-1}\right)} H_{f_{h-1}\left(e_{h-1}\right)}\right)\right] \cdot H_{f_{h}} .
\end{aligned}
$$


Now (3.5) takes the form

$$
d ! G_{i}^{(d)}=\sum_{h=2}^{d} \sum_{e} \sum_{f} \sum_{u}\left(\begin{array}{l}
d \\
e
\end{array}\right)\left(H_{i}\right)_{e, f, u}
$$

where the indices vary as follows:

$$
\begin{aligned}
& e=\left(e_{2}, \ldots, e_{h}\right), \quad 1+e_{2}+\cdots+e_{h}=d, \\
& f=\left(f_{2}, \ldots, f_{h}\right), \quad f_{j}: \mathbf{e}_{j} \rightarrow \mathbf{n}, \\
& u=\left(u_{2}, \ldots, u_{h}\right), \quad u_{j}: \mathbf{e}_{j} \rightarrow \mathbf{e}_{j-1} \quad\left(e_{1}=1\right),
\end{aligned}
$$

and

$$
\left(\begin{array}{l}
d \\
e
\end{array}\right)=\frac{d !}{e_{2} ! \cdots e_{h} !} .
$$

Note that the indices $u$ depend on $e$, but not on $f$, so that we can reverse the $u$ and $f$-summations in (4).

Now we come to the main observation of this section. A pair $(e, u)$ as above, i.e. the (finite) inverse system

$$
\mathbf{e}_{h} \stackrel{u_{h}}{\rightarrow} \mathbf{e}_{h-1} \stackrel{u_{h-1}}{\rightarrow} \cdots \stackrel{u_{3}}{\rightarrow} \mathbf{e}_{2} \stackrel{u_{2}}{\rightarrow} \mathbf{e}_{1}=\{1\}
$$

can be identified with a rooted tree $T=T_{(e, u)}$ with $d$ vertices: the set $V(T)$ of vertices of $T$ is the disjoint union of the $\mathbf{e}_{j}$ 's, the one from $\mathbf{e}_{1}$ being the "root": two vertices are joined when they correspond via one of the $u_{j}$ 's. More precisely we can label the vertices

$$
v_{j, r} \quad\left(j=1, \ldots, h ; r=1, \ldots, e_{j}\right)
$$

with $v_{1,1}$ being the root. The edges consist of all couples

$$
\left\{v_{j, r}, v_{j-1, u_{j}(r)}\right\} \quad\left(j=2, \ldots, h ; r=1, \ldots, e_{j}\right) .
$$

Thus $V(T)$ is the disjoint union of the sets

$$
V_{j}(T)=\left\{v_{j, 1}, \ldots, v_{j, e_{1}}\right\} .
$$

The latter vertices are said to be of height $j$; they are the vertices at distance (in $T$ ) $j-1$ from the root. Thus the rooted tree $T$ intrinsically determines $e_{j}=\operatorname{Card} V_{j}(T), h=$ the largest $j$ such that $e_{j}>0, e=\left(e_{2}, \ldots, e_{h}\right)$, and $d=$ Card $V(T)=e_{1}+\cdots+e_{h}$. It further determines the functions $u_{j}: V_{j}(T) \rightarrow$ $V_{j-1}(T)$ by the condition that $\left\{v, u_{j}(v)\right\}$ is required to be an edge for all $v \in V_{j}(T)$. The only element of structure in $(e, u)$ not determined by $T$ is the indexing of $V_{j}(T)$ by $1, \ldots, e_{j}$.

Now let $f=\left(f_{2}, \ldots, f_{h}\right)$ where $f_{j}: \mathbf{e}_{j} \rightarrow \mathbf{n}$ for $j=2, \ldots, h$. We can identify $f$ with an $i$-rooted labeling of $T$, i.e. with a function

$$
f: V(T) \rightarrow \mathbf{n}
$$

such that $f($ root $)=i$. Specifically, we define $f\left(v_{j, r}\right)=f_{j}(r)$ for $j=2, \ldots, h$; $r=1, \ldots, e_{j}$; and $f\left(v_{1,1}\right)=i$. Making this identification, we shall also write $f_{v}$ for $f(v)(v \in V(T))$. 
If $v \in V_{j-1}(T)$ put

(6)

$$
\begin{aligned}
v_{+} & =u_{j}^{-1}(v) \\
& =\left\{w \in V_{j}(T) \mid u_{j}(w)=v\right\} \\
& =\{w \in V(T) \mid\{w, v\} \text { is an edge and height }(w)=1+\operatorname{height}(v)\}
\end{aligned}
$$

and

$$
D_{f_{+}}=\prod_{w \in v_{+}} D_{f_{w}}
$$

Given an $i$-rooted labeling $f$ of $T$ we put

$$
P_{T, f}=\prod_{v \in V(T)}\left(D_{f_{v_{+}}} H_{f_{v}}\right) \text {. }
$$

EXAMPLE. $e_{1}=1, e_{2}=3, e_{3}=6$, and $d=1+3+6=10$.

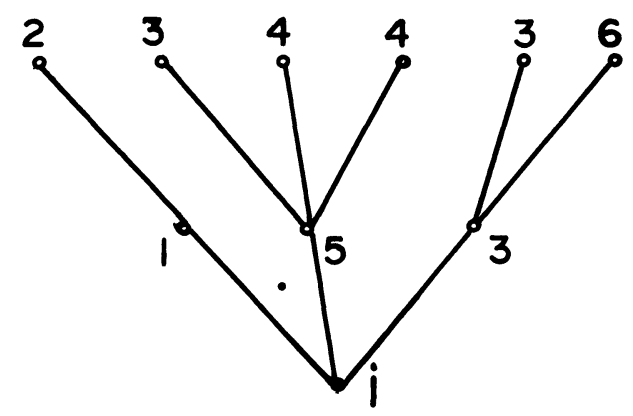

root

$$
P_{T, f}=\left(D_{1} D_{5} D_{3} H_{i}\right) \cdot\left(D_{2} H_{1}\right)\left(D_{3} D_{4}^{2} H_{5}\right) \cdot\left(D_{3} D_{6} H_{3}\right) \cdot H_{2} H_{3}^{2} H_{4}^{2} H_{6} \text {. }
$$

A review of our definitions and formula (3) shows that, for $e=\left(e_{2}, \ldots, e_{h}\right)$, $f=\left(f_{2}, \ldots, f_{h}\right), u=\left(u_{2}, \ldots, u_{h}\right)$, and $T=T_{(e, u)}$ as above, we have

$$
\left(H_{i}\right)_{e, f, u}=P_{T, f} \text {. }
$$

Thus we can rewrite (4) as

$$
d ! G_{i}^{(d)}=\sum_{h=2}^{d} \sum_{e} \sum_{u} \sum_{f}\left(\begin{array}{l}
d \\
e
\end{array}\right) P_{T_{(e, u)}, f} .
$$

We wish to write this summation over pairs $T, f$ where $T$ is a rooted tree (up to isomorphism) with $d$ vertices and $f$ is an $i$-rooted labeling of $T$ (i.e. $f: V(T) \rightarrow \mathbf{n}$ and $f($ root $)=i$ ). To do this we must, if $T=T_{(e, u)}$, insert a coefficient $C_{T}$ equal to the number of pairs $\left(e^{\prime}, u^{\prime}\right)$ for which $T_{\left(e^{\prime}, u^{\prime}\right)}$ and $T_{(e, u)}$ are isomorphic as rooted trees. As already remarked above we must have $e^{\prime}=e$. Further if $\sigma$ : $T_{\left(e, u^{\prime}\right)} \rightarrow T_{(e, u)}$ is a rooted tree isomorphism it must induce bijections $V_{j}\left(T_{\left(e, u^{\prime}\right)}\right)$ $\rightarrow V_{j}\left(T_{(e, u)}\right)$ for each $j$. The indexings then translate these bijections into permutations $\sigma_{j}$ of $\mathbf{e}_{j}$ such that the following diagram commutes. 


$\begin{array}{ccccccccc}\mathbf{e}_{h} & \stackrel{u_{h}}{\rightarrow} & \mathbf{e}_{h-1} & \rightarrow & \cdots & \overrightarrow{u_{3}} & \mathbf{e}_{2} & \stackrel{u_{2}}{\rightarrow} & \mathbf{e}_{1} \\ \sigma_{h} \downarrow & & \sigma_{h-1} \downarrow & & & & \sigma_{2} \downarrow & & \sigma_{1} \downarrow \\ \mathbf{e}_{h} & \overrightarrow{u_{h}^{\prime}} & \mathbf{e}_{h-1} & \rightarrow & \cdots & \overrightarrow{u_{3}^{\prime}} & \mathbf{e}_{2} & \overrightarrow{u_{2}^{\prime}} & \mathbf{e}_{1}\end{array}$

We can identify $\sigma$ with the element

$$
\sigma=\left(\sigma_{1}, \ldots, \sigma_{h}\right) \in S=S_{e_{1}} \times \cdots \times S_{e_{h}},
$$

where, for any $m, S_{m}$ denotes the group of permutations of $\mathbf{m}$. The group $S$ acts on the set of $u$ as above by

$$
\sigma \cdot u=\left(\sigma_{1} u_{2} \sigma_{2}^{-1}, \sigma_{2} u_{3} \sigma_{3}^{-1}, \ldots, \sigma_{h-1} u_{h} \sigma_{h}^{-1}\right) .
$$

The present discussion can be summarized by saying that $T_{\left(e^{\prime}, u^{\prime}\right)}$ is isomorphic to $T_{(e, u)}$ as a rooted tree iff $e^{\prime}=e$ and $u^{\prime}$ belongs to the $S$-orbit of $u$. Thus the coefficient $C_{T}$ we are seeking is the index [S:A] of the stabilizer $A$ in $S$ of $u$. Evidently $A$ is just the group

$$
A=\operatorname{Aut}(T)
$$

of rooted tree automorphisms of $T$. Put

$$
\alpha(T)=\operatorname{Card} \operatorname{Aut}(T) .
$$

Then $[S: A]=e ! / \alpha(T)$ where $e !=e_{2} ! \cdots e_{h} !=\operatorname{Card} S$.

With this information we can rewrite (10) as

$$
d ! G_{i}^{(d)}=\sum_{T} \sum_{f}\left(\begin{array}{l}
d \\
e
\end{array}\right) \frac{e !}{\alpha(T)} P_{T, f}
$$

where $T$ varies over rooted trees (up to isomorphism) with $d$ vertices, and $f$ varies over $i$-rooted labelings of $T$. Since $\left(\begin{array}{c}d \\ e\end{array}\right)=d ! / e$ ! we can rewrite (11), after cancellations, as follows:

$$
G_{i}^{(d)}=\sum_{T} \frac{1}{\alpha(T)} \sum_{f} P_{T, f} .
$$

This is the formula we sought. We recapitulate the result in the following theorem.

(4.1) THEOREM. Let $k$ be a commutative Q-algebra. Let $H_{1}, \ldots, H_{n} \in k^{[n]}$ be homogeneous polynomials of degree $\delta \geqslant 2$ such that $F=\left(X_{1}-H_{1}, \ldots, X_{n}-H_{n}\right)$ satisfies

$$
j(F) \quad(=\operatorname{det} J(F))=1 .
$$

Define $G_{i} \in k^{[[n]]}$ by $G_{i}(F)=X_{i}$. Then $G_{i}=\Sigma_{d \geqslant 0} G_{i}^{(d)}$, where $G_{i}^{(0)}=X_{i}, G_{i}^{(1)}$ $=H_{i}$, and for $d \geqslant 2$,

$$
G_{i}^{(d)}=\sum_{T} \frac{1}{\alpha(T)} \sum_{f} P_{T, f}
$$

Here $T$ varies over isomorphism classes of rooted trees with $d$ vertices, $\alpha(T)=$ Card Aut $(T)$, f varies over $i$-rooted labelings of $T(f: V(T) \rightarrow \mathbf{n}$ and $f($ root $)=i)$, 
and

$$
P_{T, f}=\prod_{v \in V(T)}\left(D_{f_{v_{+}}} H_{f_{v}}\right),
$$

with $v_{+}$and $D_{f_{v_{+}}}$given respectively by (6) and (7) above. $G_{i}^{(d)}$ is a homogeneous polynomial of degree $d(\delta-1)+1$, and $G_{i}$ is a polynomial iff $G_{i}^{(d)}=0$ for all $d \gg 0$.

5. Calculations. To compute further using Theorem (4.1) we introduce its generic case (with $\delta=3$ ). Explicitly, for each $d \geqslant 0$ let $\mathfrak{K}_{d}$ denote the set of monomials of degree $d$ in $X=\left(X_{1}, \ldots, X_{n}\right)$. Let $\left(C_{i, M}\right)$ be a family of indeterminates indexed by $i=1, \ldots, n$ and $M \in \mathfrak{N}_{3}$, and put $\tilde{k}=\mathbf{Q}\left[\left(C_{i, M}\right)\right]$. For each $i=1, \ldots, n$ put $H_{i}=\Sigma_{M \in \mathfrak{R}_{3}} C_{i, M} \cdot M$, a cubic homogeneous polynomial in $\tilde{k}[X]$. Let $\tilde{F}=\left(\tilde{F}_{1}, \ldots, \tilde{F}_{n}\right)$ where $\tilde{F}_{i}=X_{i}-\tilde{H}_{i}$ and

$$
j(\tilde{F})=\operatorname{det}(J(\tilde{F}))=\operatorname{det}(I-J(\tilde{H})) .
$$

We can write

$$
j(\tilde{F})-1=\sum_{d=1}^{n} \sum_{N \in \Re_{2 d}} a_{N} \cdot N,
$$

where, for $N \in \mathfrak{K}_{2 d}, a_{N}$ is a polynomial in $\left(C_{i, M}\right)$ homogeneous of degree $d$, and linear homogeneous in each of the sets of variables $\left(C_{i, M}\right)_{M \in \mathscr{N}_{3}}$ for $i=1, \ldots, n$. Thus the ideal $\mathfrak{A}$ of $\tilde{k}$ generated by all of the coefficients $a_{N}$ $\left(N \in \Re_{2 d}, d=1, \ldots, n\right)$ is (multi-) graded by degrees in each of the sets of variables $\left(C_{i, M}\right)_{M \in \mathscr{N}_{3}}$.

We put $k=\tilde{k} / \mathfrak{A}$ and denote the classes of $\tilde{H}_{i}, \tilde{F}_{i}, \tilde{F}$ modulo $\mathfrak{A}$ by $H_{i}, F_{i}, F$, respectively. Then $F=\left(F_{1}, \ldots, F_{n}\right), F_{i}=X_{i}-H_{i}$ with each $H_{i}$ cubic homogeneous in $X$, and $j(F)=1$. Moreover $F$ is generic (in an evident sense) subject to these conditions (over Q-algebras). Since $J(F)=I-J(H)$ is invertible over $k[X]$ and $J(H)$ is homogeneous of degree $2(>0)$ in $X$ it follows (Lemma (4.1) of Chapter II) that $J(H)$ is nilpotent.

To apply Theorem (4.1) we introduce the set $\mathbf{T}_{d}$ of (isomorphism classes of) rooted trees with $d$ vertices, listed below for $d \leqslant 6$.

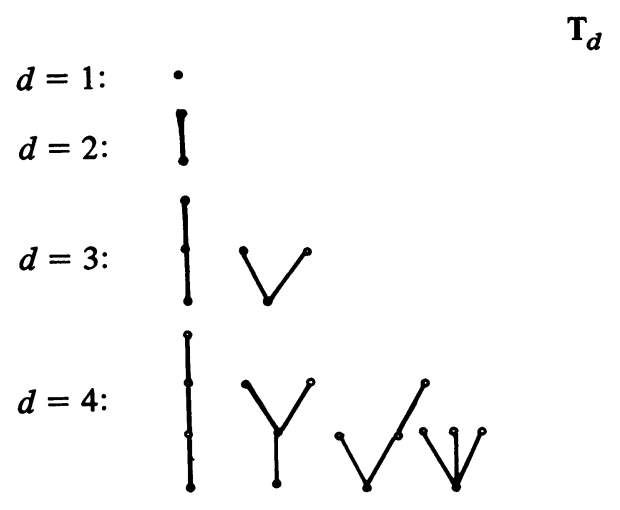




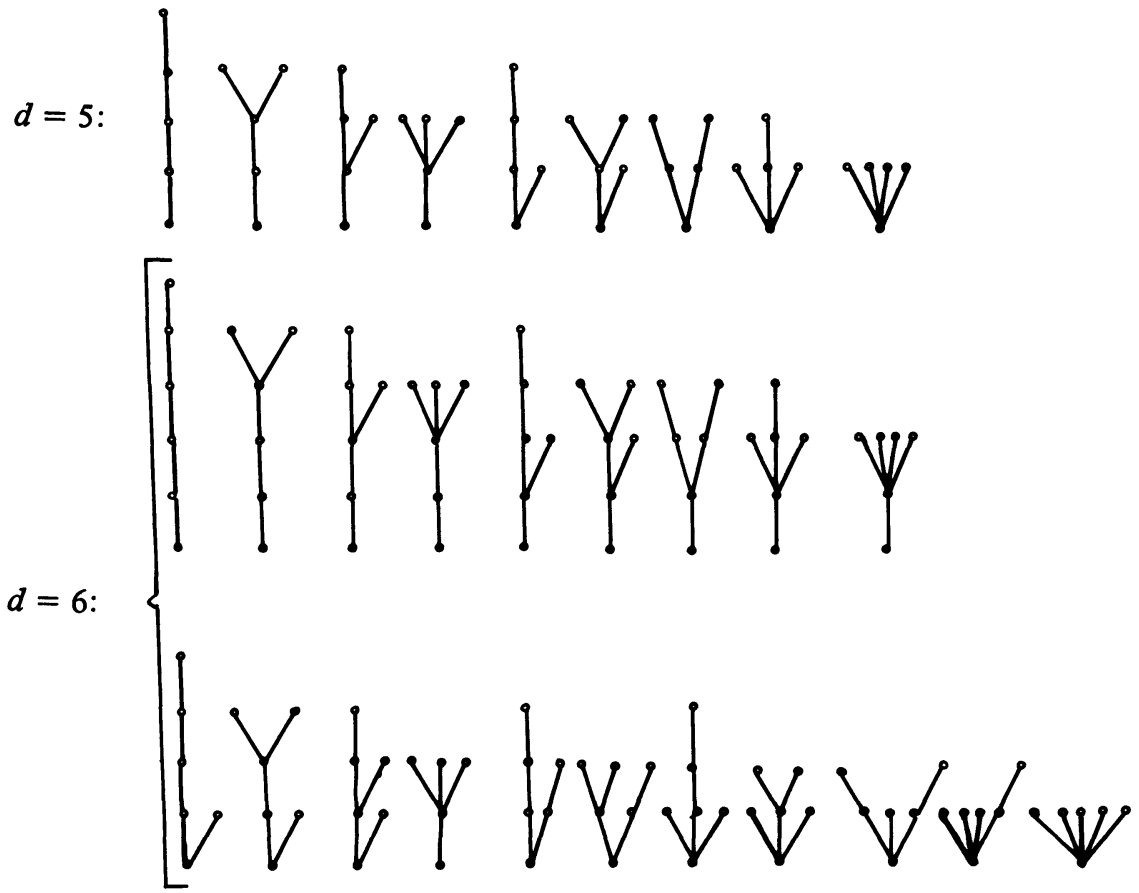

For $T \in \mathrm{T}_{d}$ and $1 \leqslant i \leqslant n$ we put

$$
\sigma_{i}(T)=\sum_{f} P_{T, f}
$$

where, as in Theorem (4.1), $f$ varies over all $i$-rooted labelings $f: V(T) \rightarrow$ $\{1, \ldots, n\}$, and where $P_{T, f}=\prod_{v \in V(T)}\left(D_{f_{v}} H_{f_{v}}\right)$.

We equip $k[X]$ with the derivations $D_{i}=\partial / \partial X_{i}(i=1, \ldots, n)$, and call an ideal $Q$ of $k[X]$ a differential ideal if $D_{i} Q \subset Q$ for all $i$. For each $e \geqslant 1$ let

$\left[J(H)^{e}\right]=$ the differential ideal of $k[X]$ generated by the entries of $J(H)^{e}$.

It is generated as an ideal by all elements $D^{p} f$ where $D^{p}=D_{1}^{p_{1}} \cdots D_{n}^{p_{n}}$ is any monomial in $D_{1}, \ldots, D_{n}$ and $f$ is any entry of $J(H)^{e}$. Clearly $\left[J(H)^{e}\right]$ contains $\left[J(H)^{e^{\prime}}\right]$ for $e^{\prime} \geqslant e$.

Given $T \in \mathbf{T}_{d}$ we shall write

$$
\sigma(T) \in\left[J(H)^{e}\right]
$$

if $\sigma_{i}(T) \in\left[J(H)^{e}\right]$ for all $i=1, \ldots, n$. (Conceivably this follows once it holds for a single $i$, because of symmetry properties among our generic $H$ 's.)

(5.1) Conjecture. Given $e \geqslant 1$, there is an integer $d(e)$ such that for all $d \geqslant d(e)$ and all $T \in \mathbf{T}_{d}$ we have $\sigma(T) \in\left[J(H)^{e}\right]$.

(5.2) RemarK. Conjecture (5.1) implies the Jacobian Conjecture.

Indeed, say we want to prove the Jacobian Conjecture over C. By Corollary (2.2) of Chapter II it suffices to do so for maps $F_{\mathbf{C}}: \mathbf{C}^{n} \rightarrow \mathbf{C}^{n}$ of the form 
$F_{\mathrm{C}}=X-H_{\mathrm{C}}$ with $H_{\mathrm{C}}$ cubic homogeneous and $J\left(H_{\mathrm{C}}\right)$ nilpotent. It follows then that $j\left(F_{\mathrm{C}}\right)=1$, so that there is a homomorphism $k \rightarrow \mathrm{C}$ such that $F_{\mathbf{C}}=\mathrm{C} \otimes_{k} F$, where $k$ and $F$ are as above. Thus it suffices to show that $F$ above is invertible. We saw above that $J(H)$ is nilpotent; say $J(H)^{e}=0$ with $e>0$. Then it follows from Conjecture (5.1) and the following formula ((12) of Theorem (4.1)),

$$
G_{i}^{(d)}=\sum_{T \in \mathbf{T}_{d}} \frac{1}{\alpha(T)} \cdot \sigma_{i}(T),
$$

that $G_{i}^{(d)}=0$ for $d \geqslant d(e)$. This shows that $G_{i}$ is a polynomial, so $F$ is invertible, as claimed.

Of course formula (3) shows that Conjecture (5.1) is stronger than necessary for the Jacobian Conjecture.

Conjecture (5.1) is trivial for $e=1$, taking $d(1)=1$. We shall present some partial results below which, in particular, imply the conjecture for $e=2$, with $d(2)=2$ (Proposition (5.3)).

Let $W_{d}=\mathbf{Z}^{\left(\mathbf{T}_{d}\right)}$, the free abelian group with basis $\mathbf{T}_{d}$, and extend $\sigma_{i}$ to a homomorphism

$$
\sigma_{i}: W_{d} \rightarrow k[X] \text {. }
$$

We extend the convention (2) to all elements $t \in W_{d}$ by writing $\sigma(t) \in\left[J(H)^{e}\right]$ if $\sigma_{i}(t) \in\left[J(H)^{e}\right]$ for $i=1, \ldots, n$.

$$
\begin{aligned}
& \text { If } T \text { contains a vertex } v \text { with } \operatorname{Card}\left(v_{+}\right) \geqslant 4 \text { then } P_{T, f}=0 \text { for } \\
& \text { every labeling } f \text { of } T \text {, and hence } \sigma_{i}(T)=0 .
\end{aligned}
$$

In fact $P_{T, f}$ contains the factor $D_{f_{v+}} H_{f_{v}}$ which, being a cubic polynomial differentiated four or more times, is zero.

In view of (4) we may restrict attention to the set $\mathbf{T}_{d}^{\prime}$ of trees $T \in \mathbf{T}_{d}$ such that $\operatorname{Card}\left(v_{+}\right) \leqslant 3$ for all $v \in V(T)$. As $d$ becomes large the trees in $\mathbf{T}_{d}^{\prime}$ must become increasingly tall. The tallest one is the straight line,

$$
\left.L_{d}=\begin{array}{l}
p \\
\vdots \\
b
\end{array}\right\} d \text { vertices, }
$$

$$
\sigma_{i}\left(L_{d}\right)=\frac{1}{3} \sum_{a=1}^{n} X_{a} \cdot\left(J(H)^{d}\right)_{i, a} \in\left[J(H)^{d}\right] .
$$

Here we write $\left(J(H)^{d}\right)_{i, a}$ for the $(i, a)$-entry of $J(H)^{d}$. Label the vertices of $L_{d}$ by $1, \ldots, d$, starting with 1 at the root. An $i$-rooted labeling $f$ of $L_{d}$ is a sequence $\left(f_{1}, \ldots, f_{d}\right)$ of integers from 1 to $n$ with $f_{1}=i$, and

$$
P_{L_{d}, f}=H_{f_{d}}\left(D_{f_{d}} H_{f_{d-1}}\right) \cdots\left(D_{f_{2}} H_{f_{1}}\right) \text {. }
$$


The $(a, b)$ entry of $J(H)$ is $J_{a, b}=D_{b} H_{a}$, so we can write

$$
\begin{aligned}
P_{L_{d}, f} & =J_{f_{1}, f_{2}} \cdots J_{f_{d-1}, f_{d}} \cdot H_{f_{d}} \\
& =\frac{1}{3} \sum_{a=1}^{n} J_{f_{1}, f_{2}} \cdots J_{f_{d-1}, f_{d}} J_{f_{d}, a} X_{a},
\end{aligned}
$$

where we have used Euler's formula for the cubic homogeneous polynomial $H_{f_{d}}$. Finally

$$
\sigma_{i}\left(L_{d}\right)=\sum_{f} P_{L_{d}, f}=\frac{1}{3} \sum_{a}\left(J^{d}\right)_{i, a} \cdot X_{a}
$$

whence (6).

To obtain further relations from (6) we introduce the following "tree surgery." Suppose that we are given $(T, v)$ and $S$, where $T \in \mathbf{T}_{d}, v \in V(T)$, and $S \in \mathbf{T}_{d^{\prime}}$. We write

$$
T_{\stackrel{0}{v} S} S
$$

for the tree in $\mathbf{T}_{d+d^{\prime}}$ obtained by joining the root of $S$ by a new edge to the vertex $v$ of $T$, and rooting the resulting tree at the root of $T$.

EXAMPLE.
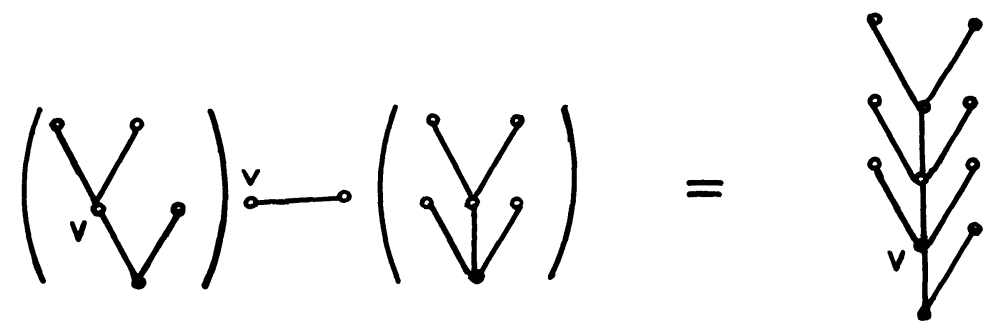

If $U$ is a third tree and $w \in V(U)$ then we have the associative law

$$
\begin{aligned}
& U_{\circ}^{w_{0}}\left(T_{\circ}^{v} \longrightarrow S\right)=\left(U_{\circ}^{w} \longrightarrow T\right)_{\circ}^{0} \longrightarrow S \text {. } \\
& \sigma_{i}(T \circ S)=\sum_{a=1}^{n} \sigma_{a}(S) \cdot \sigma_{i, v, a}(T)
\end{aligned}
$$

where $\sigma_{i, v, a}(T)$ is defined in the proof below.

An $i$-rooted labeling $f$ of

$$
T_{\stackrel{\circ}{\circ} \mathrm{S} S}
$$

consists of an $i$-rooted labeling $f_{T}$ of $T$ plus, for some $a=1, \ldots, n$, an $a$-rooted labeling $f_{S}$ of $S$. It is then clear that

$$
P_{T_{0} \multimap S, f}=P_{S, f_{S}} \cdot P_{T, f_{T}, v, a},
$$

where

$$
P_{T, f_{T}, v, a}=\left(D_{a} D_{f_{v_{+}}} H_{f_{v}}\right) \cdot \prod_{\substack{w \in V(T) \\ w \neq v}}\left(D_{f_{w_{+}}} H_{f_{w}}\right)
$$


and $v_{+}$is understood in the tree $T$, not in

\section{$T_{\circ}^{v} S$.}

Now summing over all $f$ as above we obtain (8) where $\sigma_{i, v, a}(T)=\Sigma_{g} P_{T, g, v, a}$, with $g$ ranging over all $i$-rooted labelings of $T$.

As a consequence of (8) we have:

$$
\begin{aligned}
& \text { Suppose } s=\Sigma_{r} z_{r} S_{r} \in W_{d^{\prime}}\left(\text { all } z_{r} \in \mathbf{Z}\right) \text { and } \sigma(s) \in\left[J(H)^{e}\right] \text {. } \\
& \text { Then for any }(T, v) \text { as above, putting } T_{\circ}^{*} s=\sum z_{r}\left(T \bullet S_{r}\right)
\end{aligned}
$$$$
\in W_{d+d^{\prime}} \text {, we have } \sigma\left(T_{\circ}^{v}, s\right) \in\left[J(H)^{e}\right] \text {. }
$$

If $v$ denotes the top vertex of $L_{d}$ then

$$
\sigma_{i, v, a}\left(L_{d}\right)=\left(J(H)^{d}\right)_{i, a}
$$

so, by (7),

$$
\sigma_{i}\left(L_{d^{0}{ }^{0} \longrightarrow S} S\right)=\sum_{a=1}^{n} \sigma_{a}(S) \cdot\left(J(H)^{d}\right)_{i, a} \in\left[J(H)^{d}\right] .
$$

With notation as in the proof of (6), let $f=\left(f_{1}, \ldots, f_{d}\right)$ be an $i$-rooted labeling of $L_{d}$. Then $f_{1}=i$ and

$$
\begin{aligned}
P_{L_{d}, f, v, a} & =\left(D_{a} H_{f_{d}}\right)\left(D_{f_{d}} H_{f_{d-1}}\right) \cdots\left(D_{f_{2}} H_{f_{1}}\right) \\
& =J_{f_{1}, f_{2}} \cdots J_{f_{d-1}, f_{d}} \cdot J_{f_{d}, a} .
\end{aligned}
$$

Summing over $f$ we obtain $\left(J(H)^{d}\right)_{i, a}$, whence (10).

Given $T \in \mathbf{T}_{d}$ and $S \in \mathbf{T}_{d^{\prime}}$, we define

$$
\delta_{S}(T)=\sum_{v \in V(T)} T_{0} \longrightarrow S .
$$

This belongs to the free abelian group $W_{d+d^{\prime}}$ with basis $\mathbf{T}_{d+d^{\prime}}$, and we extend it to a homomorphism

$$
\begin{aligned}
\delta: W_{d^{\prime}} \otimes W_{d} & \rightarrow W_{d+d^{\prime}}, \\
\delta(S \otimes T) & =\delta_{S}(T) .
\end{aligned}
$$

For any $s \in W_{d^{\prime}}$ and $t \in W_{d}$ we shall write $\delta_{s}(t)$ for $\delta(s \otimes t)$.

$$
\sigma_{i}\left(\delta_{S}(T)\right)=\sum_{a=1}^{n} \sigma_{a}(S) \cdot D_{a}\left(\sigma_{i}(T)\right)
$$

In view of (8) it suffices to show that

$$
\sum_{v \in V(T)} \sigma_{i, v, a}(T)=D_{a}\left(\sigma_{i}(T)\right)
$$

This, in turn, results from the fact that for any $i$-rooted labeling $f$ of $T$,

$$
D_{a}\left(P_{T, f}\right)=\sum_{v \in V(T)} P_{T, f, v, a},
$$

which is clear from the definitions. (See the proof of (8).) 
As a consequence of (12) we have

$$
\begin{aligned}
& \text { Let } s \in W_{d^{\prime}} \text { and } t \in W_{d} . \text { If } \sigma(t) \in\left[J(H)^{e}\right] \text { then } \sigma\left(\delta_{s}(t)\right) \in \\
& {\left[J(H)^{e}\right] \text {. }}
\end{aligned}
$$

Let $v$ denote the top vertex of

$$
L_{d}: \oint^{\mathrm{V}}
$$

Then if $R$ is a tree and $w \in V\left(L_{d}\right)$ we can still identify $v$ with a vertex of

$$
L_{d}{ }^{w} \longrightarrow R \text {. }
$$

We can thus define

$$
\delta_{R}\left(L_{d}\right)^{v} \longleftrightarrow S \stackrel{\text { def }}{=} \sum_{w \in V\left(L_{d}\right)}\left(L_{d}{ }^{w} \bullet R\right)^{v} \hookleftarrow S .
$$

We then have

$$
\begin{aligned}
\sigma_{i}\left(\delta_{R}\left(L_{d}\right)_{o}^{v} S\right) \\
\quad=\sum_{a=1}^{n} \sigma_{a}(S) \cdot\left(\sum_{b=1}^{n} \sigma_{b}(R) \cdot D_{b}\left(\left(J(H)^{d}\right)_{i, a}\right)\right) \in\left[J(H)^{d}\right] .
\end{aligned}
$$

Indeed it follows as in the proof of (12) that if $v \in V(T)$ then

$$
\sigma_{i, v, a}\left(\delta_{R}(T)\right)=\sum_{b=1}^{n} \sigma_{b}(R) \cdot D_{b}\left(\sigma_{i, v, a}(T)\right) .
$$

Taking $T=L_{d}$ with $v$ its top vertex and applying (10) we obtain

$$
\sigma_{i, v, a}\left(\delta_{R}\left(L_{d}\right)\right)=\sum_{b=1}^{n} \sigma_{b}(R) \cdot D_{b}\left(\left(J(H)^{d}\right)_{i, a}\right) .
$$

Now (14) follows from this and formula (8).

Let $T \in \mathbf{T}_{d}$ and let $r$ denote its root. Let $w$ be any vertex of $T$. Consider all vertices $v$ of $T$ such that any path in $T$ from $v$ to $r$ passes through $w$, i.e. all vertices "above" $w$. These form the vertices of a subtree $T(w)$ of $T$. Clearly $T(r)=T$. If $w \neq r$ then

$$
T=T^{\prime}(w)^{v} \curvearrowleft T(w),
$$

where $T^{\prime}(w)$ is the subtree spanned by the remaining vertices, and $v$ is the vertex nearest $w$ enroute from $w$ to $r$.

If $w \neq r$ we call $T(w)$ a full branch of $T$. Such $T(w)$ are precisely the subtrees $S$ of $T$ for which $T$ is of the form $T=T_{0}^{\prime v} \rightarrow S$. From the associative law (7) we see that a full branch of $S$ is also one of $T$. 


$$
\begin{aligned}
& \text { If } T \in \mathbf{T}_{d}^{\prime} \text { with } d \geqslant 2 \text { then } T \text { has a full branch } S \in \mathbf{T}_{d^{\prime}}^{\prime} \text { with } \\
& d^{\prime} \geqslant(d-1) / 3 \text {. }
\end{aligned}
$$

Recall that $\mathbf{T}_{d}^{\prime} \subset \mathbf{T}_{d}$ consists of trees such that $\operatorname{Card}\left(v_{+}\right) \leqslant 3$ for all vertices $v$, a condition obviously inherited by subtrees. Let $r$ be the root of $T$ and $t$ $(\leqslant 3)$ the number of vertices $v_{i}(i=1, \ldots, t)$ in $r_{+}$. Let $d_{i}$ be the number of vertices in $T\left(v_{i}\right)$; we may assume that $d_{1}$ is the largest. Then

$$
d=1+\sum_{i=1}^{t} d_{i} \leqslant 1+3 d_{1}
$$

so $d_{1} \geqslant(d-1) / 3$, and $T\left(v_{1}\right)=S$ is the required full branch.

Given $e \geqslant 1$, suppose that for some $d_{0} \geqslant 1$ we have $\sigma(T) \in$ $\left[J(H)^{e}\right]$ whenever $T \in \mathbf{T}_{d}$ and $d_{0} \leqslant d \leqslant 3 d_{0}-2$. Then $\sigma(T)$ $\in\left[J(H)^{e}\right]$ for all $T \in \mathbf{T}_{d}$ and all $d \geqslant d_{0}$, i.e. Conjecture (5.1) is true with $d(e)=d_{0}$.

We argue by induction on $d \geqslant d_{0}$. In view of the hypothesis we may assume $d \geqslant 3 d_{0}-1$ and, in view of (4), assume that $T \in \mathbf{T}_{d}^{\prime}$. Then, by (15), $T$ has a full branch $S \in \mathbf{T}_{d^{\prime}}^{\prime}$ with $d^{\prime} \geqslant(d-1) / 3 \geqslant d_{0}-2 / 3$, whence $d^{\prime} \geqslant d_{0}$. By assumption $\sigma_{i}(S) \in\left[J(H)^{e}\right]$. It follows therefore from (8) (or (9)) that $\sigma_{i}(T) \in$ $\left[J(H)^{e}\right]$.

(5.3) Proposition. Conjecture (5.1) is valid for $e=1$, with $d(1)=1$, and for $e=2$, with $d(2)=2$.

We treat only the case $e=2$. The case $e=1$ is trivial, or can be proved similarly.

Set $d_{0}=2$, so that $3 d_{0}-2=4$. It suffices, by (16), to show that $\sigma(T) \in$ [ $\left.J(H)^{2}\right]$ for all $T \in \mathbf{T}_{d}$ with $2 \leqslant d \leqslant 4$. The trees to be considered are

$$
L_{d}(d=2,3,4) \text {, }
$$

$$
v=q, \quad r=\{, \quad T=9, \text { and } w=99
$$

By (6) we have $\sigma\left(L_{d}\right) \in\left[J(H)^{2}\right]$ for all $d \geqslant 2$. Moreover

$$
T=L_{2} \stackrel{\text { root }}{\longrightarrow} L_{2}
$$

so also $\sigma(T) \in\left[J(H)^{2}\right]$, by (8) (or (9)).

We have $V=\delta_{L_{1}}\left(L_{2}\right)-L_{3}$, and $\sigma\left(\delta_{S}\left(L_{2}\right)\right) \in\left[J(H)^{2}\right]$ for any $S$, by (12) (or (13)). Hence $\sigma(V) \in\left[J(H)^{2}\right]$. Since

$$
Y=L_{1} \longmapsto V
$$

it follows again from (8) (or (9)) that $\sigma(Y) \in\left[J(H)^{2}\right]$. Finally, $\delta_{L_{1}}(V)=2 T+$ $W$, and $\sigma\left(\delta_{S}(V)\right) \in\left[J(H)^{2}\right]$ for any $S$, by (12). Hence $\sigma(W) \in\left[J(H)^{2}\right]$. 
(5.4) COROLLARY. If, in the setting of Theorem (4.1), we have $J(H)^{2}=0$, then $F=X-H$ is invertible with inverse $G=X+H$.

This corollary covers some of the same cases as Theorem (6.2) of Chapter II.

For $e=3$ we have tried, using the above kinds of relations (i.e. (4)-(16)) to show that,

$$
\sigma(T) \in\left[J(H)^{3}\right] \text { for all } T \in \mathbf{T}_{d},
$$

for sufficiently large $d$. For $d<7$ the calculations, though tedious, can be done by hand, and (*) does not follow (from the above relations). Dan Grayson has tested the case $d=8$ on a computer and (*) still fails.

\section{REFERENCES}

[A + K] A. Altman and S. Kleiman, Introduction to Grothendieck duality, Lecture Notes in Math., vol. 146, Springer-Verlag, Berlin and New York, 1970.

[Ab1] S. S. Abhyankar, Expansion techniques in algebraic geometry, Tata Inst. Fundamental Research, Bombay, 1977.

[Ab2] __ Lectures in algebraic geometry, Notes by Chris Christensen, Purdue Univ., 1974.

[Ab3] Historical ramblings in algebraic geometry and related algebra, Amer. Math. Monthly 83 (1976), 409-448.

$[\mathbf{A}+\mathbf{H}+\mathbf{E}] \mathbf{S}$. S. Abhyankar, W. Heinzer and P. Eakin, On the uniqueness of the coefficient ring in a polynomial ring, J. Algebra 23 (1972), 310-342.

$[\mathbf{A}+\mathbf{M}]$ S. S. Abhyankar and T.-T. Moh, Embeddings of the line in the plane, J. Reine Angew. Math. 276 (1975), 149-166.

[B] H. Bass, Algebraic K-theory, Benjamin, New York, 1968.

[B + M] S. Bochner and W. T. Martin, Several complex variables, Princeton Univ. Press, Princeton, N. J., 1948.

[C] L. A. Campbell, A condition for a polynomial map to be invertible, Math. Ann. 205 (1973), 243-248.

[C + L] I. Canals and E. Lluis, Acerca de un resultado de Segre, Anal. Inst. Matematicas, Univ. Nacional Autonoma de Mexico 10 (1970), 1-15.

[Co] E. H. Connell, A K-theory for the category of projective algebras, J. Pure Appl. Algebra 5 (1974), 281-292.

[E] W. Engel, Ein Satz über ganze Cremona Transformationen der Ebene, Math. Ann. 130 (1955), 11-19.

[F] L. Fridman, On a characterization of polynomial endomorphisms of $\mathbf{C}^{n}$, Math. USSR Izv. 7 (1973), 319-328. (Transl. from Izv, Akad. Nauk SSR 37 (1973).)

[Fu] T. Fujita, On Zariski problem, Proc. Japan Acad. 55 (1979), 106-110.

[G] I. J. Good, Generalization to several variables of Lagrange's expansion, with applications to stochastic processes, Proc. Cambridge Philos. Soc. 56 (1960), 367-380.

[Gr] W. Gröbner, Sopra un teorema di B. Segre, Atti Accad. Naz. Lincei Rend. C1. Sci. Fis. Mat. Nat. 31 (1961), 118-122. MR 24 (1962) A3155 (I. Barsotti).

[J] C. G. J. Jacobi, De resolutione aequationum per series infinitas, J. Reine Angew. Math. 6 (1830), 257-286.

[Ja] A. V. Jagžev, On Keller's problem, Siberian Math. J. 21 (1980), 141-150. (Russian)

[Jo] S. A. Joni, Lagrange inversion in higher dimension and umbral operators, Linear and Multilinear Algebra 6 (1978), 111-121.

[Ju] H. W. E. Jung, Über ganze birationale Transformatione der Ebene, J. Reine Angew. Math. 184 (1942), 161-174.

[K] O. H. Keller, Ganze Cremona-Transformationen, Monats. Math. Physik 47 (1939), 299-306.

[Ma] A. Magnus, On polynomial solutions of a differential equation, Math. Scand. 3 (1955), 255-260.

[M-L] G. Makar-Limanov, Automorphisms of a free algebra on two generators, Functional Anal. Appl. 4 (1970), 262-264 (Transl. from Funkcional Anal. i. Prilozen 4 (1970), 107-108.) 
[Mat] H. Matsumura, Commutative algebra, Benjamin, New York, 1970.

[Me] Gary H. Meisters, Jacobian problems in differential equations and algebraic geometry, Rocky Mountain J. Math. (to appear).

[Mo] T.-T. Moh, On the Jacobian Conjecture and the configuration of roots, J. Reine Angew. Math. (to appear).

[Mu] J. P. Murre, An introduction to Grothendieck's theory of the fundamental group, Tata Inst. Fundamental Research, Bombay, 1967.

$[\mathbf{N}+\mathbf{B}]$ Y. Nakai and K. Baba, A generalization of Magnus' theorem, Osaka J. Math. 14 (1977), 403-409.

[N1] Pekka Nousiainen, On the Jacobian problem in positive characteristic, Pennsylvania State Univ., preprint, 1981.

[N2] , On the degrees of smooth maps of affine space, Pennsylvania State Univ., preprint, 1981.

$[\mathbf{N}+\mathbf{S}]$ P. Nousiainen and M. Sweedler, Automorphisms of polynomial and power series rings, Cornell Univ., preprint, 1981.

[O] Susumu Oda, The Jacobian problem and the simply-connectedness of $\mathbf{A}^{n}$ over a field $k$ of characteristic zero, Osaka Univ., preprint, 1980.

[R] M. Razar, Polynomial maps with constant Jacobian, Israel J. Math. 32 (1979), 97-106.

[S] I. R. Shafarevich, On some infinite dimensional groups, Simposio Internazionale di Geometria Algebrica, 1965, published in Rendiconti di Matematica e delle sue applicazioni, Ser. 5, vol. 25, 1966, pp. 208-2121.

[Se1] B. Segre, Corrispondenze di Mobius e Transformazioni cremoniane intere, Atti della Accademia delle Scienze di Torino, Classe di Scienzo Fisiche, Mat. e Natural. 91 (1956-57), 3-19.

[Se2] , Forme differenziali e loro integrali, vol. II, Docet, Roma, 1956.

[Se3] __ Variazione continua ed omotopia in geometria algebrica, Ann. Math. Pura Appl. 100 (1960), 149-186.

[V] A. G. Vitushkin, On polynomial transformation of $\mathbf{C}^{n}$ manifolds (Tokyo, 1973), Tokyo Univ. Press, Tokyo, 1975, pp. 415-417.

[Wa] S. Wang, A jacobian criterion for separability, J. Algebra 65 (1980), 453-494.

[Wr1] D. Wright, The amalgamated free product structure of $G L_{2}\left(k\left[X_{1}, \ldots, X_{n}\right]\right)$ and the weak Jacobian Theorem for two variables, J. Pure and Appl. Algebra 12 (1978), 235-251.

[Wr2] , On the Jacobian Conjecture, Illinois J. Math. 25 (1981), 423-440.

DePartment of Mathematics, Columbia University, New York, New YoRk 10027

Department of Mathematics, University of Miami, Coral Gables, Florida 33124

DePartment of MAThematics, Washington University, St. LOUIS, Missouri 63121 